\title{
Guidelines
}

\section{German National Guideline for Treating Chronic Respiratory Failure with Invasive and Non-Invasive Ventilation: Revised Edition 2017 - Part 1}

\author{
Wolfram Windisch ${ }^{a, b}$ Jens Geiseler ${ }^{c}$ Karsten Simon ${ }^{d}$ Stephan Walterspacher ${ }^{b}$ e \\ Michael Dreher $^{f}$ on behalf of the Guideline Commission

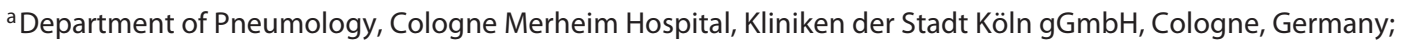 \\ ${ }^{b}$ Faculty of Health/School of Medicine, Witten/Herdecke University, Witten, Germany; ${ }^{\mathrm{C}}$ Medical Clinic IV, Pneumology, \\ Sleep Medicine and Mechanical Ventilation, Paracelsus-Klinik Marl, Marl, Germany; ${ }^{\text {d}}$ Fachkrankenhaus Kloster

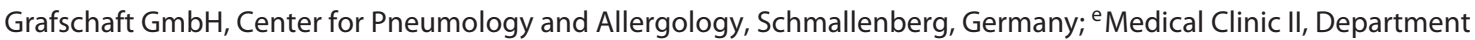 \\ of Pneumology, Cardiology and Intensive Care Medicine, Klinikum Konstanz, Konstanz, Germany; ${ }^{\text {fDivision of }}$ \\ Pneumology, University Hospital RWTH Aachen, Aachen, Germany
}

\section{Keywords}

Home mechanical ventilation - Non-Invasive ventilation . Invasive ventilation . Chronic respiratory failure - Weaning . End-of-life

\begin{abstract}
Today, invasive and non-invasive home mechanical ventilation have become a well-established treatment option. Consequently, in 2010, the German Respiratory Society (Deutsche Gesellschaft für Pneumologie und Beatmungsmedizin, DGP) has leadingly published the Guidelines on "Non-Invasive and Invasive Mechanical Ventilation for Treatment of Chronic Respiratory Failure." However, continuing technical evolutions, new scientific insights, and health care developments require an extensive revision of the Guidelines. For this reason, the updated Guidelines are now published. Thereby, the existing chapters, namely technical issues, organizational structures in Germany, qualification criteria, disease-specific recommendations including special features in pediatrics as well as ethical aspects and palliative care, have been updated according to the current literature and the health care developments in Germany. New chapters added to the
\end{abstract}

Guidelines include the topics of home mechanical ventilation in paraplegic patients and in those with failure of prolonged weaning. In the current Guidelines, different societies as well as professional and expert associations have been involved when compared to the 2010 Guidelines. Importantly, disease-specific aspects are now covered by the German Interdisciplinary Society of Home Mechanical Ventilation (DIGAB). In addition, societies and associations directly involved in the care of patients receiving home mechanical ventilation have been included in the current process. Importantly, associations responsible for decisions on costs in the health care system and patient organizations have now been involved.

(c) 2018 S. Karger AG, Basel

S. Walterspacher and M. Dreher contributed equally to this work. Guideline Commission: W. Windisch, M. Dreher, J. Geiseler, K. Siemon, J. Brambring, D. Dellweg, B. Grolle, S. Hirschfeld, T. Köhnlein, U. Mellies, S. Rosseau, B. Schönhofer, B. Schucher, A. Schütz, H. Sitter, S. Stieglitz, J. Storre, M. Winterholler, P. Young, S. Walterspacher.

This is part 1 of the German National Guideline for Treating Chronic Respiratory Failure with Invasive and Non-Invasive Ventilation - Revised Edition (Chapters 1-8). For part 2 (Chapters 9-16), see Respiration 2018, DOI: 10.1159/000488667.

\section{KARGER}

(C) 2018 S. Karger AG, Basel

E-Mail karger@karger.com

www.karger.com/res
Prof. Dr. Wolfram Windisch

Department of Pneumology, Cologne Merheim Hospital

Kliniken der Stadt Köln gGmbH, Faculty of Health/School of Medicine

Witten/Herdecke University, Ostmerheimer Strasse 200, DE-51109 Köln (Germany)

E-Mail windischw@kliniken-koeln.de 


\section{Introduction}

In November 2017, the first revision of the German "Guidelines for Non-Invasive and Invasive Home Mechanical Ventilation for Treatment of Chronic Respiratory Failure" was published [1]. The present manuscript is a direct translation of the manuscript in order to make this Guideline available to physicians outside Germany.

The use of mechanical ventilation to treat chronic respiratory failure has a long history, during which negative-pressure ventilation with the iron lung particularly became known in the first half of the 20th century. Positive-pressure ventilation now prevails in modern respiratory medicine, where it can either take place non-invasively, most often via face masks, or invasively, via tracheal cannulae, whereas non-invasive ventilation (NIV) predominates. The last 20 years have seen the publication of a large amount of research work devoted to this subject, with particular emphasis on the question of whether long-term, mostly intermittent ventilation therapy in the home setting can improve functional parameters, clinical complaints, quality of life, and long-term survival in patients with chronic respiratory insufficiency. In addition, determining the right time point at which to begin home mechanical ventilation (HMV), as well as devising optimal ventilation techniques based on scientific criteria, is also the focus of research interest. In light of this, the first national German recommendations for the implementation of mechanical ventilation therapy outside the hospital were formulated and published in 2006 [2]. The fact that the number of scientific publications on this topic and the use of ventilation therapy in a non-clinical setting have each increased over the last few years, coupled with the current discussion within the political health sector about the financial pressures on the health care system, and the need to create appropriate structures for the provision of health care, calls for the formulation of a revised scientific interdisciplinary guideline. To this end, the first version of a S2 Guideline for HMV according to the criteria of the Association of the Scientific Medical Societies in Germany (Arbeitsgemeinschaft der Wissenschaftlichen Medizinischen Fachgesellschaften e.V., AWMF) was published in 2010 [3]. The current (first) revision of the 2010 S2 Guideline has incorporated new insights gained from scientific research and taken into account the significant changes to the health care policies pertaining to patients who are ventilated in a non-clinical setting. This Guideline has been translated into English in order for the information it provides to be internationally accessible; however, since the recommendations are primarily aimed at dealing with out-of-hospital ventilated patients in Germany, it should be noted that inter-country differences in medical infrastructure prevent generalisation of these recommendations (Ch. 1.2).

\subsection{Aims of the Guideline}

The current Guideline aims to:

- Describe the specific indications (including the appropriate time point) for the initiation of HMV.

- Establish the diagnostic and therapeutic approaches required to initiate HMV.

- Establish the optimal approach for transferring the ventilated patient from the clinic into a non-clinical setting.

- Address the technical and personnel requirements for the institutes participating in the treatment of the home-ventilated patient.

- Establish a list of criteria for quality control of HMV.

- Encourage an interdisciplinary collaboration between all the professions that are involved in successful HMV therapy.

On the basis of these aims, the current Guideline has been formulated under the umbrella of the AWMF by delegated experts from the following societies and associations:

- German Respiratory Society - Deutsche Gesellschaft für Pneumologie und Beatmungsmedizin e.V. (DGP)

- German Interdisciplinary Society for Home Mechanical Ventilation - Deutsche Interdisziplinäre Gesellschaft für Außerklinische Beatmung e.V. (DIGAB)

- German Society of Anaesthesiology and Intensive Care Medicine - Deutsche Gesellschaft für Anästhesiologie und Intensivmedizin e.V. (DGAI) together with the German Association of Practising Anaesthesiologists - Kommission Niedergelassene Anästhesisten (KONA)

- Federal Association of Physicians for Chest, Sleep, and Mechanical Ventilation Medicine - Bundesverband der Pneumologen, Schlaf- und Beatmungsmediziner (BdP)

- German Interdisciplinary Society for Intensive Care and Emergency Medicine - Deutsche Interdisziplinäre Vereinigung für Intensiv- und Notfallmedizin (DIVI)

- German Society for Palliative Care Medicine-Deutsche Gesellschaft für Palliativmedizin e.V. (DGP)

- German Sleep Society - Deutsche Gesellschaft für Schlafforschung und Schlafmedizin e.V. (DGSM)

- German College of General Practitioners and Family Physicians - Deutsche Gesellschaft für Allgemeinmedizin und Familienmedizin (DEGAM) 
- German-speaking Society for Spinal Cord Injuries Deutschsprachige Medizinische Gesellschaft für Paraplegie e.V. (DMPG)

- German Society for Myopathic Diseases - Deutsche Gesellschaft für Muskelkranke e.V. (DGM)

- Federal Poliomyelitis Association - Bundesverband Poliomyelitis e.V.

- AOK Health Insurance (Northeast) - AOK Nordost

- Health Insurance Medical Service of Bavaria - Medizinischer Dienst der Krankenversicherung Bayern (MDK Bayern)

- German Industry Association - Industrieverband Spectaris

- Participation withdrawn for organisational reasons: COPD Germany - COPD - Deutschland e.V.

\subsection{Home Mechanical Ventilation Outside Germany}

The approach to HMV varies greatly across countries and regions. Comparison of epidemiological data derived from the 2005 European study "Eurovent," with survey data from Australia and New Zealand, reveal, for example, that 12 patients per 100,000 New Zealand residents and only 6.6 patients per 100,000 European residents are ventilated in a home setting $[4,5]$. Further differences can be extracted from survey data from Hong Kong (2.9 patients per 100,000 residents), Norway, Canada, and Sweden [6-9]. However, the differences both in the time points at which these data were collected, as well as the ventilation methods used, call for careful interpretation of these numbers. Nonetheless, the publication of epidemiological data from Germany would be very useful; this may be possible in the near future by evaluating the encoded medical data submitted to the insurance companies.

This obviously large variance in prevalence data can work against guidelines or recommendations. Besides the currently available German Guideline, there are some additional isolated examples of national guidelines, such as that on outpatient ventilation published by the Canadian Thoracic Society [10].

Country-specific differences become particularly apparent when the focus turns to the topics of invasive HMV, or HMV in chronic obstructive pulmonary disease (COPD) patients. The proportion of invasively ventilated patients in the home setting is around 13\% within Europe (Eurovent Data, 2005), as low as 3\% in Australia and New Zealand, and up to $50 \%$ in Poland alone $[4,5,11]$.

While the proportion of patients with HMV in Australia and New Zealand lies at 8\%, there are clearly more COPD patients in Europe (34\%) [4] and Hong Kong
(49\%) [6] who receive HMV therapy. The positive results reported by several studies on this topic has clearly led to a global change in thinking, so much so that there is now a prevailing international consensus for selected COPD patients to undergo HMV therapy [12]. These recommendations addressing the most reasonable time point at which to begin HMV in COPD patients [12] are highly congruent with the those outlined in the current Guideline. The increasing number of patients receiving HMV introduces new challenges to respiratory medicine, with modern concepts such as telemedicine shifting to the forefront. To this end, a consensus report on the subjects of HMV and telemonitoring was recently published [13].

\section{Methodology}

\subsection{Revision of the Existing S2 Guideline}

The present Guideline should be understood as an update of the "S2 Guidelines for Non-Invasive and Invasive Mechanical Ventilation for Treatment of Chronic Respiratory Failure," published in 2010 by the DGP [14]. However, it has also undergone extensive revision, with emphasis on the following features:

- Chapter reorganisation: To accommodate topics that were either excluded or only briefly considered in the first edition (e.g., spinal cord transection, secretion management, palliative medicine), either entirely new chapters were incorporated into the Guideline, or existing chapters underwent significant expansion.

- Inclusion of the latest literature.

- Contribution in part from other medical societies: It is important to note here that according to its new title given in 2010, the DIGAB follows an interdisciplinary approach and hence covers a number of areas addressed in the first edition of the Guideline. This means that the expert societies for individual specialised areas such as neurology, cardiology, and paediatrics are no longer taken into account. In turn, new expert societies and professional associations are integrated (taking into account current occupational politics as well as the new chapter composition) to fulfil this new directive.

Since 2004, the guideline classification stage S2 has been divided into the subclasses S2e (evidence-based) and S2k (consensus-based). This is partly based on what is described in the 2005/2006 edition (Domain 8, 2008) of the "German Instrument for Methodical Evaluation of Guidelines" (German: Deutsches Instrument zur methodischen Leitlinien-Bewertung, DELBI) [15]. The present Guideline strives towards the S2k classification, since a significant part deals with the scientific basis of health care, for which there are little or no evidence-based study data available. The criteria for the methodological aspects of the Guideline are as follows:

For the purposes of reaching a structured consensus, every recommendation was subjected to a neutrally moderated discussion and voting process. The goals of this were to resolve any outstanding decision problems, provide a final assessment of the recommendations, and to measure the strength of the consensus. Moderation was carried out by the AWMF. In line with the standards
68

Respiration 2018;96:66-97 DOI: $10.1159 / 000488001$
Windisch/Geiseler/Simon/Walterspacher/ Dreher 
Table 1. Chapter composition and authorship

\begin{tabular}{|c|c|c|c|c|}
\hline \multicolumn{2}{|c|}{ Chapter } & \multirow{2}{*}{$\begin{array}{l}\begin{array}{l}\text { Senior } \\
\text { author }\end{array} \\
\text { Windisch }\end{array}$} & \multirow{2}{*}{$\begin{array}{l}\begin{array}{l}\text { Leading } \\
\text { author }\end{array} \\
\text { Dreher }\end{array}$} & \multirow{2}{*}{$\begin{array}{l}\text { Coauthor } \\
\text { Siemon }\end{array}$} \\
\hline 1 & Introduction & & & \\
\hline 2 & Methodology & Windisch & Windisch & Sitter \\
\hline 3 & Scientific basis & Windisch & Stieglitz & Dreher \\
\hline 4 & Technical set-up & Siemon & Schucher & Dellweg \\
\hline 5 & Initiation, transfer, and monitoring of ventilation & Siemon & Siemon & Stieglitz \\
\hline 6 & Organisation of HMV & Dreher & Brambring & Schütz \\
\hline 7 & Nursing qualifications for HMV therapy & Dreher & Schütz & Brambring \\
\hline 8 & HMV after successful weaning & Dreher & Rosseau & Geiseler \\
\hline 9 & Obstructive respiratory diseases & Windisch & Windisch & Köhnlein \\
\hline 10 & Thoracic restrictive diseases & Windisch & Dellweg & Köhnlein \\
\hline 11 & Obesity hypoventilation syndrome & Windisch & Storre & Walterspacher \\
\hline 12 & Neuromuscular diseases & Geiseler & Winterholler & Young \\
\hline 13 & Secretion management & Geiseler & Geiseler & Schütz \\
\hline 14 & Spinal cord transection & Geiseler & Hirschfeld & Winterholler \\
\hline 15 & Special features of paediatric respiratory medicine & Geiseler & Mellies & Grolle \\
\hline \multirow[t]{2}{*}{16} & Ethical considerations and palliative medicine & Windisch & Schönhofer & Rosseau \\
\hline & Manuscript composition, figures, and layout & Windisch & Walterspacher & Sitter \\
\hline
\end{tabular}

The chapters of the guideline with the corresponding authors.

laid down by the AWMF, the recommendations were assigned the following grades: "must" (strongest recommendation), "should" (moderately strong recommendation), and "can" (weakest recommendation).

\subsection{Literature Search}

A literature search with an unrestricted publication timescale was carried out in 2015-2016 for each of the themes assigned across all of the work groups. This search was made with the aid of key search words in the databases Cochrane and PubMed/Medline. Publications in English and German were essentially considered. The resulting catalogue of relevant titles was then expanded via an informal literature search from other sources. The details of the search criteria and terminology are listed below:

Limits: Humans, Clinical Trial, Meta-Analysis, Practice Guideline, Randomized Controlled Trial, Review, All Adult: 19+ years/ Children

Mesh-term search: mechanical ventilation dren

Subgroup search: COPD, Restrictive, Duchenne, ALS, Chil-

PubMed searches using the following terms:

- Non-invasive ventilation

- Mechanical ventilation

- HMV

- Non-invasive positive pressure ventilation

- Chronic respiratory failure

- Invasive ventilation

- Tracheal ventilation

- NPPV

- NIV

- Mask ventilation

- Secretion management

- Spinal cord transection

Treating Chronic Respiratory Failure with Invasive and Non-Invasive Ventilation

\subsection{Editorial Work}

The project work for the Guideline began in the second quarter of 2015. The delegates from the DGP and the DIGAB formed work groups for each of the topics of focus (Table 1).

The text written for each key subject was edited by at least one of the leading authors and critically read by at least one of the delegated coauthors, until a consensus within the work group was achieved (Table 1). Moreover, additional corrections for each chapter took place in consultation with a representative from the editorial team (group leader).

The first ballot amongst the editorial team for the distribution of subjects and the initial manuscript composition took place in Cologne on April 15, 2015. A subsequent editorial team meeting was held on January 8, 2016, in which the single-subject manuscript drafts were critically reviewed and subsequently corrected by the authors according to protocol (Table 1). By applying the Delphi method, the delegates of the editorial team merged the single-subject manuscripts into a whole manuscript, which served as the basis for discussion at the first Consensus meeting. The outcome of this meeting was then discussed on August 26-27, 2016, in the third editorial team meeting and processed according to protocol. This was followed by the second Consensus meeting, and then the fourth editorial team meeting on December 9-10, 2016.

\subsection{Consensus Process}

The first consensus meeting took place on April 19, 2016, the second on September 27, 2016 (both in Frankfurt). The consensus meetings dealt with the balloting of the individual topic, proposals, and recommendations, and these aspects were discussed under the moderation of the AWMF (PD Dr. med. H. Sitter). Conference participants were informed about the current stage of development prior to each meeting. To this end, each participant received a copy of the updated main manuscript per email. The decision- 
Fig. 1. The respiratory system. The disorders of the respiratory systems, their implication on blood gases, and treatment.

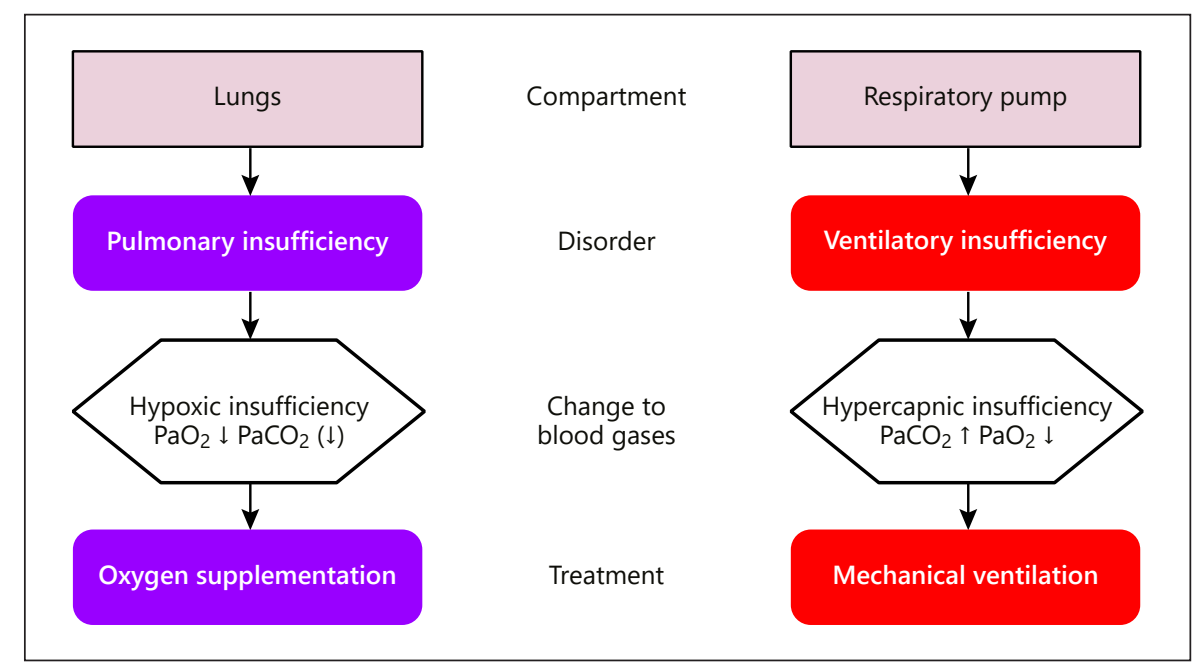

making process was subjected to the standards of a nominal group process. Accordingly, a vote took place to establish the importance of the subjects that were to be dealt with, followed by a discussion to rank these subjects in order of priority. Statements and proposals were then subjected to another round of voting, and the final voting results for the recommendations were reproduced in the manuscript with the following information: "Yes" for agreement; "No" for objection; or "Abstention." The differences in total participant numbers arise from the varying presence of participants at the consensus meetings. Any points of disagreement were extensively discussed in the setting of the consensus meeting and were ultimately reflected in the voting results. A negative voting result led to the rejection of the submitted contribution or review until a sufficient level of consensus was achieved. That way, only positive voting results were included in the Guideline revision. Complete protocols of the consensus meetings were made, and these served as a basic reference for the authors and editors involved in the review of the Guideline manuscript.

Finally, the fully edited version of the Guideline was sent to the participating medical expert societies and professional associations (but not to the industrial association Spectaris, the health insurance company representatives, or the health insurance medical service) for final assessment. A final consensus process occurred through application of the Delphi method. Based on the feedback given to the editorial team, the manuscript then went through a final round of editing and was handed in to the AWMF for verification and publication.

\subsection{Integration of Existing Guidelines}

The current Guideline is set in the context of other guidelines for mechanical ventilation therapy under the leadership of the DGP. This pertains especially to the updated S3 Guideline "NonInvasive Ventilation as Therapy for Acute Respiratory Insufficiency" [16] as well as the S2k Guideline "Prolonged Weaning" [17]. For the preparation of the current Guideline, discussion and comparison of the content with that of the other ventilation therapy guidelines took place in consultation with the respective coauthors.

\subsection{Publication}

The present Guideline was originally published in German both on the AWMF website (www.awmf.org) in July 2017, and in the journal Pneumologie for the German-speaking readership [1].

\section{Scientific Background}

\subsection{How Is Respiratory Failure Defined?}

The continuous supply of oxygen $\left(\mathrm{O}_{2}\right)$ and removal of carbon dioxide $\left(\mathrm{CO}_{2}\right)$ is essential for guaranteeing cellular metabolism in humans. The process of gas exchange within the body is ensured by the circulatory system. $\mathrm{O}_{2}$ uptake and $\mathrm{CO}_{2}$ removal take place through the respiratory system. This consists of two entirely independent components: the gas exchange system (lungs) and the ventilatory system (respiratory pump) $[18,19]$. In pulmonary insufficiency, $\mathrm{O}_{2}$ uptake is disrupted to a clinically relevant degree, while $\mathrm{CO}_{2}$ removal is not affected; the latter is due to the fact that the diffusion capacity of $\mathrm{CO}_{2}$ is over 20 times better than that of $\mathrm{O}_{2}$. In contrast, ventilatory insufficiency (respiratory pump insufficiency) involves a disruption to both $\mathrm{O}_{2}$ uptake and $\mathrm{CO}_{2}$ release. Pulmonary insufficiency is essentially treatable with $\mathrm{O}_{2}$ therapy. Severe ventilation-perfusion disorders can also require the application of positive airway pressure, in order to reopen collapsed alveoli and consequently reduce the pulmonary shunt. In contrast, ventilation therapy is necessary for hypercapnic respiratory (ventilatory) failure (Fig. 1). The combination of multiple disorders can also require the administration of oxygen in addition to ventilation therapy. 
Table 2. Signs and symptoms of chronic respiratory failure

Deterioration of the accompanying symptoms of the underlying disease (e.g., dysphagia, weight loss, dyspnoea, loss of exercise capacity)

Sleep disturbances (nocturnal awakening with dyspnoea, unrestful sleep, daytime fatigue, tendency to fall asleep, nightmares)

Erythrocytosis (polycythaemia)

Signs of $\mathrm{CO}_{2}$-associated vasodilation (conjunctive blood vessel widening, leg oedema, morning headaches)

Cyanosis

Tachypnoea

Tachycardia

Depression/anxiety/personality changes

Medical history details and clinical complaints associated with chronic respiratory failure.

The respiratory pump constitutes a complex system. Rhythmic impulses emanating from the respiratory centre are transmitted via central and peripheral nerve tracts to the neuromuscular endplates of the respiratory muscles. Contraction of the inspiratory musculature results in an increase in thoracic volume, which leads to a reduction in alveolar pressure. This, in turn, effectuates ventilation by serving as a gradient for atmospheric mouth pressure and promoting the influx of air.

Although largely dependent on the basic underlying disease, the pathophysiological consequences of ventilatory failure are usually an increased burden on, and/or reduced capacity of, the respiratory musculature, which can become overstrained as a result. Hypoventilation often first manifests during exercise and/or sleep, particularly during rapid eye movement (REM) sleep. In line with its complexity, the respiratory pump is highly vulnerable to a number of conditions, with central respiratory disorders, neuromuscular diseases (NMD), thoracic deformities, COPD, and obesity hypoventilation syndrome (OHS) serving as the main causes of respiratory insufficiency $[4,19,20]$. The aetiology of respiratory insufficiency often depends on a number of factors. With particular emphasis on COPD, there are different mechanisms associated with increased burden on the respiratory musculature (increased airway resistance, intrinsic positive end-expiratory pressure [PEEP], reduced inspiratory time, comorbidities such as cardiac insufficiency,

Treating Chronic Respiratory Failure with Invasive and Non-Invasive Ventilation anaemia) as well as the reduction in respiratory muscle capacity (volume trauma, disruption to breathing mechanics, myopathies, comorbidities such as cardiac insufficiency, diabetes mellitus) $[19,21,22]$.

Respiratory insufficiency can appear acutely and is then accompanied by respiratory acidosis. However, in the case of chronic respiratory failure, respiratory acidosis is metabolically compensated through bicarbonate retention. It is not unusual for acute respiratory deterioration to develop into chronic respiratory failure (acute to chronic). The corresponding blood gas profile is represented by a mixture of high bicarbonate levels and a degraded $\mathrm{pH}$ value.

\subsection{How Is Chronic Respiratory Failure Diagnosed?}

Medical history and clinical examination of the patient provide the first indication of the potential presence of chronic respiratory failure (Table 2 ). However, the related symptoms are so varied and non-specific [23] that the underlying disease initially stands in the foreground.

Instrument-based assessment of respiratory muscle function comprises measurement of respiratory muscle strength. Further details are available in the current recommendations published by the German Airway League (German: Deutsche Atemwegsliga) [19]. The gold standard diagnostic test for respiratory insufficiency is the determination of arterial $\mathrm{PCO}_{2}$ via blood gas analysis (BGA). In the case of sufficient circulatory perfusion, $\mathrm{PCO}_{2}$ can be assessed in capillary blood taken from the hyperemic earlobe [24]. Chronic respiratory failure is first recognised during (REM) sleep or under physical strain. An alternative to BGA is continuous transcutaneous $\mathrm{PCO}_{2}$ monitoring $\left(\mathrm{PTcCO}_{2}\right)$, which is especially advantageous for nocturnal surveillance [25]. $\mathrm{PTcCO}_{2}$ better captures the complete ventilation time course, even though individual values may deviate from those obtained by gold standard arterial BGA. It should be noted that $\mathrm{PTCCO}_{2}$ harbours a time latency of around 2 min in comparison to BGA and only delivers stable values after a preparation time of around $10 \mathrm{~min}$ [25-28]. In cases where the patient breathes ambient air, oxygen saturation (polygraphy) can, under certain circumstances, also hint at the presence of hypoventilation. However, the prerequisite for this is that the patient does not receive additional oxygen, given that this can mask even the worst cases of hypoventilation $[29,30]$.

Acute exacerbations that require hospitalisation - not uncommonly with intensive care treatment - represent the complications associated with advanced disease stages $[31,32]$. 


\subsection{How Is Ventilatory Failure Treated?}

Apart from treating the underlying disease, respiratory failure can only be treated with augmented ventilation by artificial mechanical ventilation. Acute respiratory failure requires timely initiation of mechanical ventilation, normally in an intensive care environment. Both invasive and non-invasive mechanical ventilation methods are applicable to this situation [16]. Patients with chronic respiratory failure may receive ventilation therapy at home. This is most commonly performed intermittently, usually by alternating between nocturnal ventilation and diurnal spontaneous breathing [31, 3335].

Mechanical ventilation can essentially be performed either invasively via insertion of tubes (nasotracheal, orotracheal, tracheostoma) or non-invasively. NIV can either be carried out using negative pressures (e.g., iron lung), or the now more common method of positivepressure application. Nasal masks, nasal-mouth masks, full-face masks, or mouthpieces are generally used as interfaces for NIV $[36,37]$.

\subsection{What Are the Effects of Mechanical Ventilation?}

Intermittent mechanical ventilation leads to augmented alveolar ventilation, with subsequent improvement in blood gas values both during mechanical ventilation and the spontaneous breathing interval that follows [23]. The aim of this is to achieve normalization of alveolar ventilation, whereby normocapnia serves as the benchmark. However, since both the side effects associated with the application of mechanical ventilation and the patient's acceptance of the treatment also need to be taken into account, the goal of normocapnia cannot always be achieved. While intermittent mechanical ventilation not only represents a supportive form of therapy for hypercapnic respiratory failure during the application phases, it also serves as a therapeutic measure to positively influence downstream intervals of spontaneous breathing [23]. The improvement in blood gases that is also observed during spontaneous breathing is likely to have a multifactorial basis. The main underlying mechanisms are suggested to be a resetting of $\mathrm{CO}_{2}$ chemoreceptor function in the respiratory centre, improved breathing technique, a gain in respiratory muscle strength and endurance, and the avoidance of hypoventilation during sleep $[23,38,39]$. It should be mentioned, however, that the spontaneous breathing interval in some patients becomes increasingly shorter as the disease progresses. In some cases, this can lead to a $24-\mathrm{h}$ dependency on mechanical ventilation.
Table 3. Side effects of non-invasive long-term ventilation

\begin{tabular}{lll}
\hline Side effect & $\begin{array}{l}\text { After 1 month, } \\
\%\end{array}$ & $\begin{array}{l}\text { After 12 months, } \\
\%\end{array}$ \\
\hline Dry throat & 37 & 26 \\
Facial pain & 33 & 25 \\
Fragmented sleep & 27 & 20 \\
Impaired nasal breathing & 22 & 24 \\
Abdominal bloating & 22 & 13 \\
Flatulence & 19 & 17 \\
Sleep impairment & 13 & 16 \\
Eye irritation & 12 & 11 \\
Nasal bleeding & 7 & 2 \\
Nausea & 1 & 2 \\
Facial pressure sores & 1 & 0 \\
Vomiting & 0 & 0 \\
\hline
\end{tabular}

Adapted from [42].

The improvement in alveolar ventilation induced by mechanical ventilation has ensuing effects, of which the most prominent are subjective alleviation of the abovedescribed symptoms, as well as the improvement in health-related quality of life [40-42]. The latter is understood as a multidimensional psychological construct that characterises the subjective condition of the patient on at least 4 levels, namely physical, mental, social, and functional. Assessment of health-related quality of life in scientific studies is dominated by questionnaires in which disease-specific measuring tools can be distinguished: the Severe Respiratory Insufficiency Questionnaire (SRI) [41, $43,44]$ has been developed for the specific measurement of quality of life in patients undergoing HMV therapy. This questionnaire and the tools for its evaluation are freely available in various languages for download and usage from the DGP website (www.pneumologie.de).

\subsection{What Are the Side Effects Associated with Mechanical Ventilation?}

Standing in opposition to the positive physiological and clinical effects of long-term mechanical ventilation are the side effects induced through either the ventilation interface or the ventilation therapy itself (Table 3). The most significant problems associated with invasive mechanical ventilation are barotrauma, volume trauma, infections, tracheal injuries, bleeding, wound granulation tissue, stenoses, fistula formation, cannula occlusion and/ or displacement, dysphagia, dysarthria, pain, and impaired coughing. 


\section{Technical Set-Up}

Mechanical ventilation represents a treatment that strongly encroaches on the integrity of the patient, but is also often life-sustaining. Besides quality management of the ventilation therapy, an autonomous lifestyle for the patient has the highest priority. The respiratory physician determines the indication for therapy, selects the ventilation machine and ventilatory mode, sets the ventilation parameters, and ultimately holds clinical responsibility for all these aspects (see Ch. 5).

Unsupervised alterations to the ventilator can lead to potentially life-threatening complications. Changes to the ventilation system or settings should therefore only take place following the physician's orders and must be performed under supervision. The following areas warrant special mention:

- Ventilation machine

- Ventilation interface

- Expiratory system

- Oxygen application system, location, and rate

- Humidification system

- Ventilation parameters

All those involved with the use of the ventilation system (patient, relatives, nursing staff, other carers) should undergo an authorised training session for each piece of equipment. The basic requirements for ventilation devices are regulated according to ISO standards, which distinguish between "Home Mechanical Ventilation Machines for Ventilator-Dependent Patients"(DIN EN ISO 806012-72:2015 [45]) and "Home Mechanical Ventilation Machines for Respiratory Support" (DIN EN ISO 10651-6: 2011 [46]). The service life of the various consumable materials is established by the manufacturer, although this is generally not evidence-based.

The basic technical requirements for the ventilation devices are regulated in accordance with the corresponding EU standards $[45,46]$. Patients who are not dependent on mechanical ventilation can also be supplied with a machine from a category of those usually reserved for ventilatordependent patients; that is, the associated therapy quality and acceptance level are proven to be better. This, however, does not apply vice versa. A second ventilation machine and an external battery are required for ventilation times $\geq 16 \mathrm{~h}$ per $24 \mathrm{~h}$. In exceptional cases, a second ventilator may be necessary due to other reasons [47] (e.g., in mobile patients using a ventilator attached to a wheelchair). In all cases, the ventilation machines should be identical.

A ventilation machine with an internal battery is necessary both for its use in patients with life-sustaining me-

Treating Chronic Respiratory Failure with Invasive and Non-Invasive Ventilation chanical ventilation and for patients who are unable to remove the mask by themselves [45]. When spontaneous breathing ability is strongly reduced (daily ventilation time $\geq 16 \mathrm{~h}$ ), an external battery with sufficient capacity is necessary [47].

\subsection{Non-Invasive Ventilation}

NIV is mostly carried out intermittently but can also be used in patients who are completely dependent on mechanical ventilation. The necessary technical equipment depends on the underlying disease and level of dependency.

\subsubsection{Alarms}

In NIV, ventilation masks that are not completely sealed can frequently lead to leakages, at least intermittently. Although leakages can be clinically insignificant, the machine alarms that they elicit can lead to a significant impairment of sleep quality and quality of life. In patients who are not dependent on mechanical ventilation, silencing of alarms (apart from the power failure alarm) should therefore be made possible.

It can be helpful to save both the alarm and parameter settings, since ventilation parameters in the home environment can either be consciously or subconsciously altered from the physician's original instructions [48, 49]. In ventilator-dependent patients, the disconnect alarm should not have the capacity for deactivation. Alarm management is a special technical feature of mouthpiece ventilation. Connection to an external alarm system should be available as an option for patients on life-sustaining mechanical ventilation.

\subsubsection{Tubing}

Single-tube systems with a corresponding exhalation system are most commonly used. Double-lumen tube systems are only necessary in NIV when expiratory volume needs to be reliably determined; however, this is rarely the case. Single-use systems should be replaced in cases of contamination or defects.

\subsubsection{Expiratory System}

Open-outlet expiratory systems and controlled exhalation valve systems can be fundamentally distinguished (Table 4). Controlled exhalation valves can be integrated into the ventilator when a double-lumen tube system is in place. In single-tube systems, the valve needs to be positioned close to the patient. Exhalation valves have different exhalation resistance levels and characteristics, meaning that ventilation should be assessed upon change-over, since this may lead to dynamic volume trauma in some 
Table 4. Comparison of different expiratory systems in non-invasive ventilation

\begin{tabular}{ll}
\hline Controlled valve & Open system \\
\hline Can be used without PEEP & Higher peak pressure due to mandatory PEEP application \\
\hline Loud noise during expiration & Quieter system \\
\hline Lower trigger sensitivity & Better trigger sensitivity \\
\hline Additional control tube potential source of error & $\begin{array}{l}\text { Continuous airflow from openings can potentially cause irritation } \\
\text { to eyes and face }\end{array}$ \\
\hline Elimination of $\mathrm{CO}_{2}$ dependent on tidal volume and dead space & $\begin{array}{l}\text { Elimination of } \mathrm{CO}_{2} \text { dependent on PEEP and position of air } \\
\text { discharge points }\end{array}$ \\
\hline No loss of oxygen through the valve during inspiration & \begin{tabular}{l} 
Increased oxygen demand due to higher wash-out \\
\hline Hybrid modes not available
\end{tabular} \\
\hline
\end{tabular}

diseases [50]. In the open or so-called leakage systems, defined openings in the ventilation system adjacent to the patient (as an insert in the tubing or mask) serve to wash out expired $\mathrm{CO}_{2}$. Continuous positive pressure PEEP or expiratory positive airway pressure [EPAP]) should be applied, since a notable amount of $\mathrm{CO}_{2}$ can otherwise be rebreathed from the tubing system [51]. The position and type of exhalation openings also contribute to the efficacy of $\mathrm{CO}_{2}$ elimination, which should be clinically tested. Different open-outlet systems cannot be switched without clinical assessment of the quality of mechanical ventilation $[52,53]$. In the case of severe hypoxemia, the use of an open-valve system is associated with a markedly lower concentration of inhaled oxygen fraction compared to a controlled valve system; therefore, the latter system is preferable in severe hypoxemia [54].

\subsubsection{Ventilation Modes}

There is no standard nomenclature available for the ventilation modes. In the last few years, a series of new ventilation modes have appeared that present as composites of the existing modes and can hence be described as hybrid modes, adding confusion to the field [55].

\subsubsection{Negative-versus Positive-Pressure Ventilation}

Nowadays, positive pressure is by far the most common mode of mechanical ventilation, with little scientific evidence to support the long-term effects of negativepressure ventilation [56]. The latter can, however, be used in special cases, such as in children, or when problems with positive-pressure ventilation cannot be resolved.

\subsubsection{Pressure versus Volume Preset}

Pressure-preset mechanical ventilation essentially provides the possibility to compensate for leakage [57,
58]. Randomised cross-over studies investigating the effectiveness of nocturnal NIV showed no differences between the use of pressure versus volume presets in terms of relevant physiological and clinical outcome parameters [59-61]. However, the use of pressure-preset mechanical ventilation was associated with fewer side effects [60]. If the patient shows signs of deterioration or failure under a particular ventilation mode, an alternative ventilation mode can be attempted under inpatient conditions [62].

4.1.4.3 Hybrid Modes, Automated Modes, etc.

The prerequisite for hybrid mode application is the use of leakage systems $[63,64]$. There are still no long-term data available for the clinical advantages of ventilation modes combining pressure and volume presets. For OHS patients in particular, the application of ventilation with pressure and volume presets (target volume) led to an improvement in nocturnal ventilation, but not in sleep quality or quality of life [65-70].

The effects of hybrid mode ventilation on COPD have thus far been established solely as non-inferior [61, 71]. Subjective sleep quality in COPD patients was found to be better than that under standard ventilation therapy with high pressures and frequencies [70]. Hybrid mode ventilation can be applied if the physiological parameters for breathing and sleep patterns show a verified improvement, or if tolerance for this particular mode is subjectively better. Therapy adherence generally tends to be better using hybrid modes [72]. A range of highly different, manufacturer-specific technical systems are available; an overview of these systems was recently published [73].

\subsubsection{Assisted versus Assisted-Controlled Mode}

Choosing between these ventilation modes depends on the underlying disease and its level of severity, ventila- 
Table 5. Comparison of the different types of masks

\begin{tabular}{llll}
\hline & $\begin{array}{l}\text { Commercial } \\
\text { nasal masks }\end{array}$ & $\begin{array}{l}\text { Commercial } \\
\text { oronasal masks }\end{array}$ & $\begin{array}{l}\text { Customised } \\
\text { masks }\end{array}$ \\
\hline $\begin{array}{l}\text { Dead space } \\
\text { Oral leakage }\end{array}$ & Small & $\begin{array}{l}\text { Larger } \\
\text { Likely }\end{array}$ & $\begin{array}{l}\text { Unlikely } \\
\text { Pressure ulcer risk }\end{array}$ \\
Cost & $\begin{array}{l}\text { Medium } \\
\text { Lower }\end{array}$ & $\begin{array}{l}\text { Higher } \\
\text { Lower }\end{array}$ & $\begin{array}{l}\text { Optimised } \\
\text { Higher }\end{array}$ \\
\hline
\end{tabular}

Type-specific characteristics of different mask types for non-invasive ventilation. tion set-up, and patient tolerance. Prospective, randomised long-term studies comparing the different forms of ventilation are lacking. In one randomised cross-over study in patients with COPD and hypercapnia, pressuresupported ventilation and pressure-controlled ventilation with high respiratory rates, both using high inspiratory pressures, were equivalent over a period of 6 weeks [74]. Recent studies in COPD patients have demonstrated the superiority of ventilation therapy that aims to effectively reduce $\mathrm{CO}_{2}[23,60,75-78]$.

\subsubsection{Triggers}

Trigger sensitivities (inspiratory and expiratory trigger) differ significantly amongst individual ventilation machines and may influence ventilation quality; this particularly pertains to synchronisation between the patient and the ventilator [79-81]. In patients with COPD, expiratory fluctuations in airflow frequently lead to trigger errors; an early expiratory trigger suspension period may therefore be helpful in individual cases.

\subsubsection{Pressure Build-Up and Release}

Individual settings for the speed of pressure build-up and breakdown can support efficiency and patient tolerance. This should be adjusted according to the aetiology of the respiratory failure.

\subsubsection{Ventilation Interfaces}

In principle, nasal masks, oronasal masks, face masks, mouth masks, and mouthpieces are available $[55,82]$ (Table 5). The ventilation helmet is not suitable for HMV use.

In general, nasal masks offer greater patient comfort $[83,84]$ and for physical reasons cause fewer problems with pressure ulcers, but often carry the problem of oral leakage during sleep [85]. This, in turn, can negatively influence ventilation and sleep quality [82, 86-88]. An oronasal mask can ameliorate this $[89,90]$; however, the use of oronasal masks in patients with obstructive sleep apnoea may lead to a deterioration in nocturnal sleep patterns [91]. If the oronasal mask is not tolerated by the pa- tient, the application of a chin band can be useful in individual cases $[88,90]$. Since the mask represents the fundamental connection between the ventilator and the patient, the patient's preferences should be taken into consideration [84]. When an oronasal mask or chin band cannot be tolerated, the use of humidification can lead to an improvement [92]. Current real-life data from COPD patients show that in contrast to earlier years, oronasal masks are the preferred type of ventilation interface, and the frequency of use of these masks compared to nasal masks has increased in parallel with inspiratory pressure application [93].

Full-face masks can serve as a supplement or an alternative to existing ventilation masks in the event of problems with pressure ulcers. Mouth masks are an alternative to nasal ventilation, especially when ventilation times are relatively long and the skin pressure points on the nose require relief $[94,95]$. Ventilation via a mouthpiece is particularly useful in high-dependency NMD patients $[94,96]$.

While commercial masks often suffice, customised masks can become necessary if high ventilation pressures and long ventilation times are applied, if the ready-made mask fits poorly, or if the patient has sensitive skin. Custom-made masks are also useful for patients with NMD, or with the inability to independently adjust the position of the mask, especially since long ventilation times are often necessary. Since the range of commercial masks for paediatric patients is much more limited, customised masks are more frequently required in this patient group. A further advantage of these masks is their minimal dead space with a better reduction in $\mathrm{CO}_{2}[53,97]$; however, this rarely serves as an indication in practice. Readjustment of the mask can also be necessary at short intervals (e.g., with a change in body weight, musculature, or skin turgor).

Every patient should have a spare mask at hand. In patients who normally use a customised mask, the replace- 
ment mask can be a commercial one, provided it fits adequately. Multiple masks/mouthpieces may be necessary for long periods of ventilation in order to avoid pressure ulcers.

\subsubsection{Humidification and Warming}

Supplemental humidification is not normally required for NIV. However, some patients experience clinically relevant dehydration of the mucosal membranes with an accompanying increase in nasal resistance $[92,98]$. The need for humidification is based on the patient's symptoms [82] as well as on the presence of insufficient ventilation quality with increased nasal resistance.

Inspiratory air-conditioning systems can be basically distinguished as active or passive. Active humidifying systems exhibit very different performance characteristics [99]. Humidifiers in which the air passes through water (bubble-through humidifiers) can theoretically generate infectious aerosols if the water is contaminated. This is not the case, however, with humidifiers in which the air passes over the surface of the water (pass-over humidifiers), hence the reason why sterilised water should be renounced upon [100]. It should be noted, however, that the study by Wenzel et al. [100] was performed under continuous positive airway pressure (CPAP) conditions.

Passive humidifying systems (heat and moisture exchangers, HMEs) conserve the patient's own humidity and airway temperature [101]; therefore, they are less effective in ventilation with leakage [102]. Humidifying systems show no indicatory differences physiologically; however, patients have reported a subjective preference for active humidification [103]. Therapeutic decisions should therefore take place on an individual basis.

\subsubsection{Inhalation Treatment}

The effectiveness of administering inhalable medication during mask ventilation depends upon many factors, with ventilation pressure, the exhalation system [104], and the place at which the medication is administered all exerting an influence $[105,106]$. In principle, pulmonary deposition is better during spontaneous breathing than under NIV, providing the correct inhalation manoeuver is performed. Therefore, inhalation therapy should only occur under NIV if there are signs of insufficiency under spontaneous breathing [107].

\subsubsection{Pulse Oximetry}

The use of a pulse oximeter to monitor HMV is normally not necessary, except in patients with NMD and coughing insufficiency. In this case, a drop in oxygen sat- uration can be an early sign of imminent, clinically significant mucus retention that requires special measures for cough support (Ch. 12, 13) [108]. Continuous pulse oximetry is sometimes necessary in children (Ch. 15).

Recommendations for Non-Invasive Ventilation

- Home access to non-invasive ventilation must be granted after considering the technical advantages and disadvantages, the individual patient tolerance levels, and the results of clinical testing.

- Commercial masks are usually sufficient. Customised masks must only be used under exceptional circumstances such as in neuromuscular disease patients, with high ventilation pressures or long ventilation times, or in patients with sensitive skin.

- Every patient should have a spare mask.

- Hybrid or special ventilation modes such as mandatory target volume cannot be recommended on a general basis.

- A second ventilation machine and an external battery are necessary for ventilation times $\geq 16 \mathrm{~h} / 24 \mathrm{~h}$.

\subsection{Invasive Ventilation}

\subsubsection{Alarms}

In contrast to NIV, the use of an alarm system for invasive ventilation is mandatory. Disconnection and hypoventilation alarms should be present. Lasting deactivation of these alarms can pose a considerable threat to the patient and should therefore not be technically possible. Invasive ventilation systems with speaking mode (speaking valve, unblocked cannulae, cannulae with fenestrations) require an alarm management feature that recognises the disconnect and hypoventilation statuses in any case.

\subsubsection{Tracheostoma}

A tracheostoma should be stable in order to be able to proceed with HMV therapy; therefore, an epithelialised tracheostoma should be surgically created in elective tracheotomy for HMV. Due to shrinkage tendencies and the risk of incorrect cannula positioning, dilational tracheotomies are acceptable only after showing evidence of sufficient stability after longer periods of cannula insertion. Cannula replacement in a percutaneous dilational tracheostoma can safely be carried out by a specially trained nursing team alone. The safety benefits gained from surgical conversion of a previously placed dilational tracheostoma need to be weighed up against the risk and burden of the operative procedure, particularly in patients with multiple morbidities who have undergone unsuccessful prolonged weaning [17]. 


\subsubsection{Tracheal Cannulae}

Ventilation via tracheal cannulae can usually be carried out with either blocked or unblocked cannulae [109]. A cuff pressure gauge is required for blocked cannula use. Here, the measuring range should be able to reliably represent the recommended maximum cuff pressure of 30 $\mathrm{cm} \mathrm{H}_{2} \mathrm{O}$ [110]. In addition to the required spare cannulae of the same size, smaller cannulae should always be at hand in order to facilitate an emergency cannulisation if cannula changeover becomes difficult [47].

Provided there is intact laryngeal function, the use of a speaking valve can enable the patient to speak whilst under mechanical ventilation therapy [111-113]. Here, the use of a cuffless cannula, a completely unblocked cannula, or a fenestrated cannula is mandatory. The application of PEEP leads to improved speaking ability [114]. Nevertheless, the cannula-induced relocation of the tracheal lumen can amount to dynamic volume trauma of the lungs.

\subsubsection{Secretion Management}

Invasively ventilated patients require an aspirator, which should essentially be a powerful one ( $\geq 25 \mathrm{~L} / \mathrm{min}$ ). Aspiration should be as atraumatic as possible; a flat endotracheal tube is usually sufficient for this purpose [115]. A replacement aspirator is necessary. One device should have the option of battery power to guarantee aspiration in the event of a power failure, or during movement. The maximal diameter of the aspiration catheter should be half the inner diameter of the tracheal cannula [115].

\subsubsection{Humidification and Warming}

During invasive ventilation, conditioning (i.e., humidification and warming) of the inspired air prevents both the bronchial mucosa from drying out and the secretions from thickening [116]; therefore, it is constantly required. Conditioning can be carried out either through HME filters or via active humidification, with or without tubal heating. HME filters are simpler to use, but can have unfavourable effects on the work of breathing, breathing mechanics, and $\mathrm{CO}_{2}$ elimination $[117,118]$.

The application of HME filters is insufficient or ineffective when using speaking valves, unblocked cannulae, or fenestrated tracheal cannulae with air escape via the larynx, thus requiring active humidification. The latter, however, can markedly impede mobility, making it sometimes necessary to alternate between the two. Simultaneous use has to be avoided, so as to prevent a marked increase in HME filter resistance.

\subsubsection{Inhalers}

A particle size that is as small as possible $(1-3 \mu \mathrm{m})$ is critically important for lower airway deposition [119121]. Since warm-air humidification reduces deposition [121-123], the applied dosage should be doubled [124]. For aerosol generation, both nebulizers that are approved for HMV machines as well as metered-dose inhalers (MDIs)/soft mist inhalers (SMIs), can be used [125, 126].

Various adapters are available for MDIs/SMIs [127]. Optimal deposition can be achieved with a spacer system through which the airflow passes [122, 128-130]. In contrast, adapters with side ports (e.g., elbow adapters) are associated with markedly lower deposition rates [131]. This does not hold true for the use of SMIs [132]. The MDI/SMI triggering time point is critical and should occur towards the end of the expiration phase $[122,125]$.

\subsubsection{Pulse Oximetry}

A pulse oximeter is necessary for selective measurement of oxygen saturation during invasive ventilation; continuous monitoring of oxygen saturation may be necessary in certain disease groups (paediatrics, spinal cord transection, incapacitated patients). Pulse oximeters should conform to the norm for electric medical devices if they are used for surveillance [133].

Finger pulse oximeters can be used for selective measurements (e.g., during mucus management procedures). Pulse oximetry is not appropriate for the reliable detection of hypoventilation; therefore, its application is only useful after appropriate training [134-136].

\subsubsection{Other Accessories for Invasive Ventilation}

A ventilation bag with an oxygen connection port for use in tracheal cannulae and masks is required [47].

\section{Recommendations for Invasive Ventilation}

- The tracheostoma should be surgically created; proof of stability is absolutely necessary for percutaneous dilational tracheostomata.

- A second ventilation machine and an external battery are essential for ventilation times $\geq 16 \mathrm{~h} / 24 \mathrm{~h}$.

- A pulse oximeter is necessary for selective measurements and possibly for continuous measurements in certain disease groups (i.e., spinal cord transection, paediatrics).

- A small-diameter spare cannula must be made available.

- For application of speaking valves, a cuffless cannula, a completely unblocked cannula, or a fenestrated cannula must be used.

- Conditioning (humidification and moistening) of the ventilation air is mandatory.

Two aspirator devices are necessary. 


\subsection{Non-Invasive and Invasive Ventilation}

\subsubsection{Ventilation Parameters}

The ventilation machine should be chosen so that a lasting or even temporary decline in the patient's ventilatory function can be sufficiently dealt with. Ventilators differ considerably from one another in terms of trigger properties, pressure stability, pressure build-up, etc., so that even a formally equivalent set-up can result in clinically significant differences in ventilation [57, 79-81, 137-141]. Exchanging a ventilation machine for another type or switching the ventilation mode should therefore take place during surveillance in hospital. Alteration of the ventilation parameters is only permitted under the responsibility of a physician with sufficient ventilation expertise.

\subsubsection{Oxygen Admixtures}

The addition of oxygen should follow the individual technical guidelines for each ventilator. This is possible either through an inlet on the machine, or via an adapter within the ventilation tubing system. According to EU norms [45], if the ventilation device harbours a corresponding function for oxygen administration, the oxygen fraction should be measured; in all other cases the measurement of the oxygen fraction is not necessary [45]. The oxygen flow rate is clinically titrated. An alteration to the feed-in location also changes the effective oxygen influx. In an open system, the required oxygen flow rate is generally higher than that in a system with a controlled expiratory valve [54, 142-146]. Flow-controlled demand oxygen systems are not suitable for use as an oxygen source during mechanical ventilation [147].

\subsubsection{Additional Functions}

Usage statistics and pressure/flow records stored within the machine are useful for assessing therapy quality and adherence [69].

\subsubsection{Particle Filters}

Machine-adjacent particle filters in the air inlet vicinity are necessary. Filters in the outlet vicinity of the machine are mandatory for hospital use. A definitive statement regarding particle filter use in HMV is not possible due to a lack of relevant studies. The shelf life stated by the manufacturer is very short and based on measurements from invasively ventilated patients in an intensive care setting. Studies on intubated and ventilated patients show that particle filter shelf lives of up to 1 week are possible, without risk to the patient [148]. A change-over interval of 1-7 days is feasible, depending on the status of the patient.

\subsubsection{Hygienic Preparation of Devices}

Before reinstating a ventilation machine previously used by another patient, hygienic preparation of the device according to the manufacturer's instructions is mandatory.

\subsubsection{Capnometry}

Measurement of end-expiratory $\mathrm{CO}_{2}$ is mentioned in the valid EU norms as a means of monitoring expiration in ventilation machines for ventilation-dependent patients [45]. The correlation between $\mathrm{PtcCO}_{2}$ and $\mathrm{PaCO}_{2}$ in restrictive diseases is markedly better than that in parenchymal lung diseases. Since the results of end-expiratory $\mathrm{CO}_{2}$ measurements in patients with pulmonary diseases do not reliably correlate with $\mathrm{PaCO}_{2}$, their use in adult patients with diseased lungs is generally not advisable $[27,149]$. Spinal cord transection patients represent an exception (Ch. 14). Measurements should be performed using the main airflow mode. Regular use of transcutaneous $\mathrm{CO}_{2}$ devices at the current technical standard is rarely indicated for HMV use, although transcutaneous measurements are reliable from a technical standpoint [150].

\subsubsection{Aids for Coughing Insufficiency}

For more details, see Chapter 13.

\section{Initiation, Adaptation, and Control of Home Mechanical Ventilation}

\subsection{What Are Ventilation Centres?}

There is currently no clear definition of a ventilation centre. This Guideline distinguishes between a weaning centre and an HMV centre (expertise in NIV or invasive HMV) (Fig. 2). "Ventilation centre" is used as an umbrella term (with different lines of focus). The following definitions deliberately refrain from naming professional groups. The most significant specialist disciplines are quoted as examples.

The following section semantically distinguishes between three different types of ventilation centres:

I. Weaning centre (Ch. 8.5)

II. HMV centre with expertise in invasive HMV

III. HMV centre (with special focus on NIV)

Patients who are either undergoing or have undergone prolonged weaning are primarily cared for at the weaning centre; this can also include patients undergoing invasive HMV. The primary purpose of HMV centres (Group III) is the initiation of mechanical ventilation, mainly non-
Windisch/Geiseler/Simon/Walterspacher/ Dreher 
Fig. 2. Definitions of "weaning centre" and "home mechanical ventilation centre."

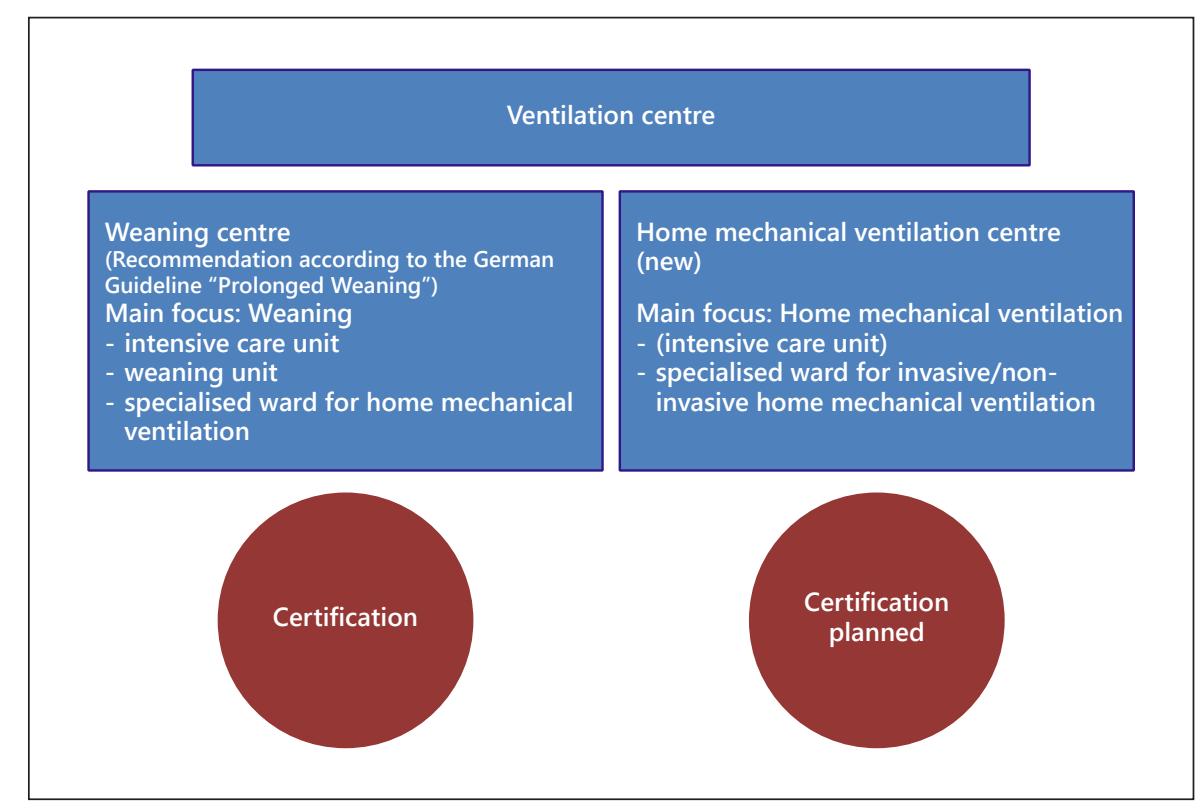

invasive, and in some cases, invasive (mainly elective, but also under emergency conditions); Group II centres have an additional focus on the initiation and monitoring of patients receiving invasive HMV. In the future, the certification of centres for HMV should be implemented on an occupational policy level.

\subsubsection{What Are the Tasks of a Home Mechanical \\ Ventilation Centre?}

HMV should be organised by a HMV centre. The home-ventilated patient requires this centre for the setup, monitoring, and optimisation of the ventilation therapy, for emergency admission in cases of deterioration, and as a contact partner for the home nursing team and treating general practitioner [151].

Failed weaning (Group 3c) (Ch. 8.1; Table 6) [152] that requires continuous/intermittent invasive HMV should be attested by the weaning centre, where HMV should also be initiated. If relocation of the patient for this purpose is not feasible, initiation of HMV should take place in close coordination with the weaning centre.

\subsubsection{What Sort of Expertise Is Required in a Home}

Mechanical Ventilation Centre?

Given the complexity of the patient's multiple morbidities, it should be possible at any time to integrate the therapeutic expertise from other relevant specialty disciplines (e.g., palliative medicine, neurology, or paediatrics) with that of a specialized respiratory physician for mechanical ventilation. Close consultation across the various disciplines needs to take place. Expertise in HMV is indispensable. The machine-specific requirements arise from the diagnostic necessities for initiation or monitoring of ventilation, as follows.

\subsection{Where Should the Initial Set-Up Take Place?}

HMV should initially be set up in a HMV centre. Details about unsuccessfully weaned patients who require continuous/intermittent invasive HMV therapy can be found in Chapter 8. Disease-specific indication criteria will be described in detail in later chapters.

\subsubsection{Which Basic Diagnostic Tests Are Necessary?}

The initial basic clinical diagnosis consists of general and respiratory failure-specific history-taking as well as a physical examination. The following (technical) tests are also essential:

- Electrocardiogram (ECG)

- Diurnal and nocturnal BGA under room-air conditions, or in the case of long-term oxygen therapy, with the prescribed oxygen flow rate

- Lung function tests (spirometry, whole-body plethysmography, respiratory muscle function tests, if applicable [e.g., $\mathrm{P}_{0.1}, \mathrm{P}_{\text {Imax }}$ ])

- Basic laboratory tests

- X-ray of the thorax, with consultation of earlier X-ray images if necessary

- Polygraphy, polysomnography (if necessary)

- Exercise testing (e.g., 6-min walking test) 
Table 6. Weaning categories

\begin{tabular}{|c|c|c|}
\hline Group & Category & Definition \\
\hline 1 & Simple weaning & Successful weaning after the first SBT and first extubation \\
\hline 3 & Prolonged weaning & $\begin{array}{l}\text { Successful weaning only after at least } 3 \text { unsuccessful SBTs or ventilation for longer than } \\
7 \text { days after the first unsuccessful SBT }\end{array}$ \\
\hline $3 \mathrm{~b}$ & $\begin{array}{l}\text { Prolonged weaning with } \\
\text { subsequent NIV }\end{array}$ & $\begin{array}{l}\text { Successful extubation/decannulation only after at least } 3 \text { unsuccessful SBTs, or } \\
\text { ventilation for longer than } 7 \text { days after the first unsuccessful SBT and only through the } \\
\text { use of NIV, potentially with the continuation of NIV as HMV }\end{array}$ \\
\hline
\end{tabular}

Further tests are quite often necessary for special or unclear disease profiles and should therefore be made available:

- Continuous nocturnal $\mathrm{CO}_{2}$ measurements (e.g., transcutaneous $\left.\left[\mathrm{PtcCO}_{2}\right]\right)$ [27]

- Assessment of effective coughing (Ch. 13)

- Vital capacity (VC) measurements in sitting and supine positions

- ECG, in cases where there is historical or clinical evidence for left or right heart insufficiency, coronary disease, or heart defects

- Ultrasound of the diaphragm [153]

- Endoscopic procedures (laryngoscopy/bronchoscopy)

- Fibreoptic endoscopic evaluation of swallowing [154] Non-volitional tests for respiratory muscle strength are desirable but are not always available [19]. Similarly, neurophysiological examination of the diaphragm and phrenic nerve should be aimed for in special cases as an alternative to ultrasound $[155,156]$. For such cases, the enlistment of neurological/neurophysiological expertise should take place early on, and collaboration with a neuromuscular clinic should be sought.

\subsubsection{How Is Ventilation Therapy Implemented?}

\subsubsection{Who Sets Up the Ventilation?}

Therapy initiation takes place under the responsibility of a physician; it can also be delegated to respiratory therapists, specialist nurses, or other specially trained health care professionals. The physician does not necessarily have to be on site, provided appropriate communication aids are available (telephone, telemonitoring). The indication for HMV initiation and the selection of the ventilation equipment (machine, masks, tubes, etc.) are the responsibility of the physician or the HMV centre and depend on the underlying illness. Independent set-up by employees from equipment provider companies should be declined. The overall responsibility for this process lies with the physician. Telemedicine consultations with a physician could be used in the future, like that already being established in intensive care medicine [157]; however, research on how this should be implemented is still lacking [13].

\subsubsection{What Needs to Be Observed during the Setting-}

\section{Up Process?}

The extent of surveillance required during the initiation period depends on the underlying disease and its severity. A very high initial $\mathrm{PCO}_{2}$ reading can particularly lead to rapid entry into sleep with REM rebound, which in the event of technical ventilation problems such as ventilation tube disconnection may lead to deleterious hypoventilation. Pulse and blood pressure monitoring, oximetry, and/or $\mathrm{PtcCO}_{2}$ assessment, and measurement of tidal volume (optimally with optical presentation of the ventilation graphs) are additional means by which the efficacy of respiratory muscle unloading can be estimated.

5.2.2.3 Which Examinations Are Necessary for Initiation of Mechanical Ventilation?

During the course of the initiation process, ventilation efficacy can be measured via the determination of $\mathrm{PCO}_{2}$
Windisch/Geiseler/Simon/Walterspacher/ Dreher 
under spontaneous breathing conditions and ventilation, and can be supplemented with nocturnal measurements. The following procedures are suitable for nocturnal ventilation assessment, depending on the symptom profile of the patient:

- $\mathrm{PtcCO}_{2}$

- Polygraphy/pulse oximetry

- Polysomnography

- Spot check analysis of blood gases

Normal oxygen saturation under ambient air conditions does not rule out the presence of hypoventilation. The parameter that best depicts the quality of ventilation is $\mathrm{PCO}_{2}[31,158,159]$. The gold standard for $\mathrm{CO}_{2}$ measurement is BGA and hence $\mathrm{PaCO}_{2}$. The withdrawal of blood for BGA can, however, disturb the patient's sleep and thereby potentially reduce the $\mathrm{CO}_{2}$ reading. The $\mathrm{PtcCO}_{2}$ measurement offers the advantage of estimating $\mathrm{CO}_{2}$ values in a non-invasive and continuous fashion. However, in the absence of calibration with $\mathrm{PaCO}_{2}$, it does not provide reliable absolute values, so that the relative changes have to be referred to as diagnostic criteria [27]. The end-tidal $\mathrm{CO}_{2}$ measurement is problematic, since the true $\mathrm{PaCO}_{2}$ value can be underestimated due to leakages (especially under NIV therapy) or ventilationperfusion disorders [27, 31, 160, 161].

5.2.2.4 What Are the Aims of Mechanical Ventilation?

The aim of mechanical ventilation is to improve the patient's symptoms and, in turn, quality of life. Increased life expectancy is achieved for many clinical disorders. These goals are achieved through the treatment of alveolar hypoventilation (respiratory pump insufficiency) and hence through a decrease in subsequently elevated $\mathrm{PaCO}_{2}$.

\subsubsection{How Are These Aims Achieved?}

These goals are achieved through the sufficient augmentation of alveolar ventilation, whereby, depending on the underlying disease, the inspiratory pressure levels have a large range $[60,76,77]$. Respiratory muscle unloading under NIV therapy is defined by the degree of unloading (ventilation quality) and the duration of NIV use (quantity) within $24 \mathrm{~h}$. Overall, unloading should ideally lead to the normalisation, or at least reduction, of $\mathrm{PCO}_{2}$ during the day. Although diurnal ventilation is usually effective [162], nocturnal NIV is preferable. A combination of nocturnal and diurnal ventilation can be prescribed for pronounced overloading of the respiratory muscles [163]. Whether controlled ventilation exclusively leads to maximal $\mathrm{CO}_{2}$ reduction in every patient is unclear, since assisted ventilation procedures also have therapeutic effects and can lead to $\mathrm{CO}_{2}$ reduction $[77,80$, 164].

Treating Chronic Respiratory Failure with Invasive and Non-Invasive Ventilation

\subsubsection{Is Oxygen Supplementation Necessary?}

Additional oxygen application is indicated if oxygen saturation $\left(\mathrm{SpO}_{2}\right)$ remains below $90 \%$ despite optimal ventilation with sufficiently high ventilation pressure and the elimination of hypoventilation, or if the partial pressure of arterial oxygen $\left(\mathrm{PaO}_{2}\right)$ is $<55 \mathrm{~mm} \mathrm{Hg}$ during therapy [165]. The disease-specific features of mechanical ventilation initiation will be discussed in the relevant chapters below.

\subsection{What Sort of Check-Ups Are Necessary and at}

Which Time Intervals Should They Occur?

There are no scientific data to indicate how often a home-ventilated patient should undergo check-ups. According to opinions published in the literature, the suggested check-up intervals range from a few weeks to up to 1 year $[166,167]$. Given that it often takes time to adapt to HMV in the initial phase, this Guideline recommends that the first check-up with nocturnal diagnostics occurs within the first $4-8$ weeks of implementing NIV [168, 169].

Repeated check-ups can be useful in the event of poor therapy adherence; if therapy efficacy is still lacking despite optimal therapy set-up, mechanical ventilation should be ceased. This decision is solely the responsibility of the treating physician in the mechanical ventilation centre. In principle, check-ups are recommended at least 1-2 times per year, depending on the type, degree of stability, and progression of the underlying disease, as well as the quality of the settings thus far. Shorter check-up intervals may be necessary in cases where there is rapid progression of the underlying disease.

\subsection{Where and How Should These Check-Ups Be Carried Out?}

HMV check-ups are essentially carried out in hospital, with a focus on nocturnal monitoring. However, since the public health care system is currently undergoing a transition, a multisector approach to HMV patient care is generally preferable. To this end, the question of whether follow-up of HMV patients is possible in an outpatient setting is currently being discussed. This offers the possibility for HMV patients to either undergo check-ups in an NIV outpatient centre situated within HMV centres, or for the check-ups to occur in the home environment through a physician with relevant expertise. However, relevant scientific data are lacking. Therefore, in the name of patient safety, scientific research on this topic is urgently required. The current Guideline additionally refers to pilot projects that are already up and running (for more 
information, see www.digab.de). A general recommendation for outpatient check-ups cannot, however, be formulated in the current Guideline.

In addition to the tests outlined in Chapter 5.2.1, the check-up examination should be supplemented by the following:

- Adherence to therapy

- Assessment of the clinical success of ventilation

- Side effects of the ventilation (e.g., mask issues, nasal mucosa problems)

- Inspection of the ventilation system (e.g., ventilation set-up, humidification unit, ventilation interface, accessories)

- Screening of nocturnal ventilation efficacy

Notwithstanding are the technical check-ups in the context of medical product legislation, which should essentially be carried out by the manufacturer.

\subsection{What Needs to Be Noted for the Exchange of a}

Ventilation Device or Interface?

The exchange of structurally equivalent ventilation devices with retainment of all parameters can take place in a non-clinical setting. However, different machines even from the same manufacturer - are not equivalent $[80,138,168,170]$ (Ch. 4) and should therefore be exchanged under controlled conditions during a hospital stay or in an HMV centre, to avoid compromising the quality of the mechanical ventilation therapy.

Switching between tracheal cannula models [171] and ventilation masks [89] can also substantially influence ventilation quality and should therefore only occur in collaboration with an HMV centre or a hospital (if necessary). Changing the type of tracheal cannula should be carried out with the assistance of a local endoscopic examination to avoid abnormal positioning with early and long-term tracheal complications.

\subsection{Deterioration of Health}

Measures that should be undertaken in the event of acute deterioration of the patient's health status should be discussed with all those involved in the ongoing care process. This plan of action should contain the necessary steps for approaching emergency situations (positions for facilitating respiration, oxygen administration, cannula exchange, secretion management, medication [e.g., antibiotics], emergency calls, etc.), but should also be oriented towards the patient's wishes and stipulations. Ideally, all other measures that are either desired or not wished for by the patient should be laid down in an advanced health care directive [172-179].
Recommendations

- Establishment of home mechanical ventilation must take place in a home mechanical ventilation centre.

- Ventilation therapy must improve the symptoms of hypoventilation by way of $\mathrm{PCO}_{2}$ reduction.

- The criteria for supplementary long-term oxygen therapy should be reviewed once the best possible ventilation set-up has been achieved.

- The first ventilation check-up must occur soon after ventilation establishment and evaluate treatment success on the basis of subjective, clinical, and technically measureable parameters.

- Modification of ventilation (ventilation set-up, ventilation interface) must only be carried out under the instruction of a physician.

- Structurally equivalent devices can be exchanged with identical settings. Structurally different devices must be exchanged under controlled conditions.

- Endoscopic identification of the correct tracheal cannula location must take place when switching to a different tracheal cannula model.

\section{Organisation of Home Mechanical Ventilation}

Mechanical ventilation therapy outside the hospital should meet the medical requirements and respect the needs and wishes of the patient in question. To this end, the extent of care as well as the provision of medical aids should be adapted to meet the demands of the patient's individual disease profile and capabilities. Factors that should especially be noted here are ventilation duration and ventilation interface as well as the involvement of relatives (if applicable). This is only possible through a multidisciplinary, multisector collaboration between all those participating in the therapy. By way of example, the documented discrepancies between ventilation centre regulations versus those ascertained in the home setting should be avoided $[49,180]$.

The organisation of HMV is strongly dependent on the chosen type of approach to therapy. While patients without motoric limitations can largely accomplish the mostly nocturnal NIV themselves, those with physical disabilities potentially require care that ranges from extensive support to the constant presence of a carer for lifesustaining continuous NIV. In principle, the requirements for establishing invasive HMV are even more complex. This especially pertains to managing the transition from the hospital to the home $[17,181]$ as well as to the skill and competency of the assigned nursing team. To this end, the recommendations for specialised nursing care (Ch. 7) and the particularities of the patient observation process (Ch. 6.8) should especially be noted. 
6.1 Which Forms of Care Are Available for Home Mechanical Ventilation Patients and How Do They Differ from One Another?

The following living options are available to those receiving HMV therapy:

- At home: either completely autonomously, or with the support of a specialised outpatient nursing service or personal assistant.

- Outpatient-run shared apartments for people undergoing ventilation therapy, or living communities in an intensive care setting.

- Inpatient nursing facilities with an emphasis on HMV care.

Thereby, the following types of care can be distinguished:

- Assisted care: Untrained aids, lay helpers, assistants, or relatives can generally serve as care supporters, without the involvement of trained nursing staff. The main priority is directed towards providing support for an autonomous lifestyle for the patient. Assisted care can be used in the realm of the employer model/personal budget, can be carried out by relatives, or can be realised through a combination of nursing services and employer models (Ch. 7.7, 7.8).

- Specialised nursing care: This form of care is ensured through an outpatient nursing service or an inpatient nursing care facility using qualified nursing staff (Ch. 7).

The decision about whether assisted or specialised care should be carried out either in the patient's home, in a shared living community, or in an inpatient nursing facility lies with the patient itself, or with the patient's legal guardian following clarification and advice from the leading clinician $[182,183]$. The type of outpatient-run home and care facility selected can additionally depend on the severity of the disease, the complexity of care required, the structural requirements (accessibility), and the regional availability of outpatient services.

The leading clinician is responsible for the organisation of outpatient medical and nursing care up to the point where the care of the patient is taken over by the treating physician (general practitioner and/or other specialists; see Ch. 6.6) in an outpatient sector. The patient is allowed to leave the hospital after the establishment of HMV only if the provision and funding of subsequent care can be fully guaranteed. The more dependent the patient is on the ventilator and the reduction in autonomy that results, the more extensive the transition into the outpatient care sector is [17], meaning the whole process can potentially take weeks.

Treating Chronic Respiratory Failure with Invasive and Non-Invasive Ventilation
Recommendation

- With respect to quality of life and perspectives, the appropriate living arrangements (own home, shared living, or inpatient nursing facility) as well as the proper type of care (independent living vs. assisted or specialised nursing care) should be determined in consultation with the patient and, next to his/her medical requirements, be oriented towards the patient's wishes and needs.

\subsection{Which Tasks Are Undertaken by the Home}

Mechanical Ventilation Centre before and after

Discharging the Patient?

Upon discharge from the hospital, the patient becomes "home ventilated." Ideally, the discharge process is organised by a HMV centre (Ch. 5.1), which determines the time point for patient discharge in consultation with those involved in HMV care. Following patient discharge, the HMV centre remains available on a consultation basis for patients and relatives, as well as for the treating physician, nursing staff, participating therapists, and the ventilation equipment provider. Furthermore, the ventilation centre also carries out regular patient check-ups. For emergency care services, regional hospitals that are close to the patient's living quarters should be integrated into the care program.

Recommendation

- Home mechanical ventilation should be initiated at a home mechanical ventilation centre with relevant expertise and should be organised by such a centre following discharge from hospital (Ch. 5).

\subsection{What Needs to Be Observed before a Ventilated \\ Patient Is Discharged into the Home Mechanical Ventilation Sector?}

The transition from the hospital to the outpatient sector represents a vulnerable phase [49], especially for invasively mechanically ventilated patients. The prerequisites for discharge from hospital are:

- Verifiably tested option for switching to NIV in patients who have undergone successful weaning following intensive care therapy (Weaning Category $3 \mathrm{c}$ ), particularly in the case of intermittent ventilation (Ch. 8.1).

- Stable underlying disease and comorbidities [184].

- Stable ventilation status without complications using the same ventilation machine as that intended for home use.

- Stable tracheostoma. For safety reasons, an epithelialised tracheostoma is preferable for outpatient care. In the case of a percutaneous dilatative tracheostoma, it should be demonstrated that safe cannula exchange can be performed by the nursing staff alone (Ch. 4.2.2). 
- Permitted take-over of costs for nursing care and medical aid supplies.

- Supply with all necessary devices, consumables, and materials, and availability of these aids at the time of discharge.

- Appointment for the first follow-up examination at the HMV centre (Ch. 5.1).

If the patient has not yet reached his/her own optimal level of functional efficiency and performance, (early) rehabilitative measures should be undertaken prior to release into the outpatient sector [183].

\section{Quality Indicators}

- In patients with weaning failure (Weaning Category $3 \mathrm{c}$ ), the change-over to non-invasive ventilation has been tested and the lack of success documented in the patient's records.

- Prior to discharge from hospital, the patient has been transferred to the ventilation machine that corresponds to the one intended for home mechanical ventilation, with appropriate and validated adjustment of the ventilation parameters.

- The appointment for the first check-up examination at the home mechanical ventilation centre has been arranged prior to discharge and noted in the patient's medical report.

\section{Recommendations}

- The patient must be in a stable state prior to hospital discharge.

- The cost transfer for nursing care and supply of medical aids must be guaranteed by the clinic prior to discharge.

- Post-hospital care in a home mechanical ventilation centre must be organised prior to discharging the patient.

\subsection{Managing the Transfer of a Patient to a Home \\ Mechanical Ventilation Setting Can Present as a \\ Challenge: How Is This Organised?}

In Germany, the complex hospital discharge process for transfer to the outpatient ventilation sector falls within the realm of responsibilities of the hospital from which the patient is being discharged (see Social Insurance Code $\$ 39$ Sozialgesetzbuch [SGB] V). Outpatient care of the ventilated patient should be completely organised before discharge from hospital, ideally through a multidisciplinary transfer management team. The transfer management team should collaborate closely with the patient or legal guardian, as well as the caregivers and/or relatives, and consist of the following professionals [183]:

- Coordination transfer manager (e.g., physician, respiratory therapist or case manager with extensive experience in HMV)

- Treating physicians (in- and outpatient)

- Nursing team (in- and outpatient)

- Equipment provider

- Social workers, social education specialists (if needed)
- Therapists (if needed)

- Funding bodies

During the transfer process, which usually takes $2-3$ weeks [17] - or potentially longer under certain circumstances - early arrangement of the definitive date of discharge is particularly important for further coordination of the various personnel required, and for guaranteeing the outpatient care service [183]. Hereby, the patient is entitled to an independent and a comprehensive, neutral consultation, combined with the provision of information about relevant self-help organisations, since discussions with patients can help them to accept their situation and structure their daily routine. The consultation should not be directly related to the transfer management process, but should instead in advance provide an overview of the various types of living and care arrangements available. The use of checklists is recommended for the highly complex transfer process; such assessment tools can be requested from the DIGAB or the medical service of health insurance companies.

Listed below are the minimum requirements for a proper transfer operation, which should be processed before discharge from hospital, specified in the medical report, and given to the patient as a copy before he/she leaves the clinic. Checklists for assisting the transfer process should be oriented towards these minimum requirements:

- Diagnoses and therapy goals

- Post-hospital medication including pro re nata (PRN) medication

- Information about the medication administered before discharge from hospital, particularly sedatives, analgesics, and antibiotics

- Screening test results for multidrug-resistant pathogens (MRP)

- Recommendations and time intervals for clinical follow-up examinations, including arrangement of the first check-up appointment at the HMV centre

- Proposed living and care arrangements

- Extent of care (nursing staff attendance times)

- Time frame and content of nursing provisions

- Technical set-up of ventilation and monitoring, including accessories (Ch. 4)

- Type of ventilation interface; cleaning and changeover intervals

- Ventilation mode, with specification of all parameters

- Duration of mechanical ventilation and potential spontaneous breathing phases

- Oxygen flow rate during mechanical ventilation and spontaneous breathing phases 
- Measures for secretion management

- Application of inhalable medication

- Planning of dietary requirements

- Information about the social environment that will contribute to the patient's care

- Psychosocial support for the patient and relatives (if necessary)

- Arrangement of training sessions for patient and relatives

- Additional resources (e.g., wheeled walker, toilet seat raiser, nursing bed, communication aids, consumables, continence items, wound management, etc.)

Quality Indicator

- Upon discharge from hospital, the patient receives a medical report with the above-specified information.

Recommendations

- Outpatient care of the ventilated patient must be completely organised before discharge from hospital.

- The discharge process must be organised under the responsibility of the treating clinic by a transition management team, with the assistance of a checklist.

- The patient or the legal guardian of the patient must be comprehensively informed (e.g., through the use of relevant information sheets) about the potential care options available.

\subsection{Who Is Involved in the Provision of Home-Based Care?}

The following professionals should contribute to the outpatient care of a ventilated patient:

- Physicians (general practitioners and/or other specialists)

- If applicable, an outpatient nursing team (specialised/ assisted; see Ch. 6.1, 7)

- Equipment provider for the configuration of the prescribed medical devices and their technical maintenance

- If applicable, an outpatient therapeutic team (speech therapists, occupational therapists, physiotherapists, social therapists, educators, psychologists)

- Funding providers

\subsection{What Kind of Demands Are Placed on the}

Treating Physician in the Home Mechanical

Ventilation Sector?

The outpatient treating physician carries the responsibility for the outpatient medical treatment of a patient receiving HMV therapy. Knowledge about the expected course of the disease, the prognosis, and the nursing and therapeutic measures required is necessary for the physician to be able to professionally advise the patient and clearly formulate the goals of the mechanical ventilation therapy. The physician should therefore be able to demonstrate experience in out-of-hospital ventilation and should carry out home visits. With the relevant qualifications, this task can be taken on, for example, by a general practitioner, similarly by a respiratory specialist, an anaesthetist, a paediatrician, a neurologist, an internal medicine specialist, or a specialist with extra training in intensive care medicine and hence mechanical ventilation. If the required medical expertise is not available, the supervising HMV centre should become involved on an advisory basis. Medical care can also be generally undertaken by a team of physicians from different disciplines that correspond to the disease profile of the patient. In the future, the multisector medical care network should be supported by intersectoral telemedicine procedures such as teleconsultations.

\section{Recommendations}

- The outpatient treating physician must undertake the responsibility of outpatient treatment of a patient receiving home mechanical ventilation therapy.

- The supervising home mechanical ventilation centre must become involved where necessary and be available for advice.

\subsection{What Kind of Demands Are Placed on the Nursing}

Team in the Home Mechanical Ventilation Sector?

Due to the particular challenge and undertaking of a wide-reaching responsibility, the demands placed on the nursing service are described in detail in Chapter 7.

\subsection{What Sort of Requirements Exist for Equipment \\ Providers in Relation to the Supply of Ventilation Aids?}

The equipment provider is responsible for the initial briefing on the ventilation machine and its entire set of accessories (e.g., oxygen supply, respiratory gas humidifiers) as well as the monitoring devices. Furthermore, the equipment provider serves as the primary contact for technical problems. An authorised equipment provider must be appropriately qualified.

The initial briefing should take into account the current versions of the guidelines outlined in the Medical Products Law (German: Medizinproduktegesetz, MPG) and the Medical Products Operator Ordinance (German: Medizinprodukte-Betreiberverordnung, MPBetreibV). Functional checks, maintenance intervals, and safety-related inspections should be carried out in accordance with the manufacturer's suggestions. The commissioned equipment provider should guarantee round-the-clock availability and provide a prompt, needs-based service 
$[183,185,186]$. It should be possible to resolve technical problems with the ventilation device within $24 \mathrm{~h}$.

Notifiable matters must be reported both to the safety representative of the company supplying the device and the Federal Institute of Pharmaceuticals and Medical Products (German: Bundesinstitut für Arzneimittel und Medizinprodukte, BfArM). Such matters include functional defects, breakdowns, changes in device features or performance, and any incorrect labelling or description on the product or its operating manual that could directly or indirectly lead to the death or serious deterioration in the health status of the patient, the device operator, or any other person.

\section{Recommendation}

- The equipment provider must carry out an initial briefing on the ventilation device in accordance with medical product laws and guarantee permanent availability with a prompt, needs-based service.

\subsection{What Sort of Need Exists for Therapeutic Services and What Sort of Demands Are Placed on the} Therapists?

Supplemental therapies are medical services prescribed by accredited physicians and performed by specialised therapists. These include the following:

- Physical therapy

- Voice, speech, and language therapy

- Occupational therapy

The prescription of therapeutic services is regulated by the Therapy Guideline of the Joint Federal Committee (German: Heilmittel-Richtlinie des Gemeinsamen Bundesausschusses). Outpatient physiotherapy, speech therapy, and occupational therapy services can encourage patient autonomy, achieve symptom relief and, in some circumstances, promote weaning potential. Thereby, continuation of the required therapies in the outpatient setting should already be established during the transition phase from the clinic, whilst taking into account the regional circumstances and availability of therapeutic services. Hereby, prompt continuation of therapy should be aimed for. Prescription takes place through the treating physician.

Focus on the following areas of therapy is useful for HMV patients:

- Occupation therapy, which operates on a functional or sensorimotor-perceptive level.

- Targeted speech, voice, and swallowing therapy in patients with tracheal cannulae as well as in those at risk of aspiration.
- Physiotherapy in the context of secretion management as well as manual therapy with respect to physiological motility and further neurophysiological and pneumological treatment procedures.

Under certain conditions, it may be necessary for the therapists to carry out home visits. The treating therapist should have proven experience in the treatment of mechanically ventilated and tracheotomised patients. If this is not the case, acquisition of an applicable supplementary qualification is recommended. Relevant courses are currently in development, such as the "Specialised therapist for ventilation" advanced training course (German: Fachtherapeuten für Ventilation) offered by the DIGAB.

The therapist is obliged to demonstrate familiarity with each ventilation device if he/she is working independently with the ventilated patients, without the presence of a qualified nurse. In addition, skills in the areas of endotracheal suctioning, tracheal cannula management, oxygen application, and emergency response are required in order to be able to react accordingly in specific cases of need. These special features require evidence for the completion of a relevant advanced training course in compliance with the medical delegation responsibility.

Recommendation

- Physiotherapy, speech therapy, and occupational therapy should, depending on the relevant indications, form an inherent part of the outpatient treatment program for home-ventilated patients, and be prescribed by the treating physician at a suitable intensity level.

\subsection{What Especially Needs to Be Observed for the}

Surveillance and Documentation of Home Mechanical Ventilation?

In cases of either life-sustaining NIV or invasive ventilation in an outpatient setting, the ventilation parameters (configured target parameter) and ventilation readings (measured current values) as well as the alarm limits must be continuously monitored and documented at least once during each shift. Changes to ventilation readings must not just be documented, but in some circumstances may also require a causal analysis and, if needed, a direct and relevant response to the issue.

Ventilation parameters and alarm limits are set according to the physician's instructions; in this respect, the establishment of individual, needs-based alarm limits should receive special attention. The replacement ventilation device (if available) should also be checked once per shift for function and accuracy of the current ventilation parameters. The replacement device should be within reach of the patient, properly functioning, preset, and on
86

Respiration 2018;96:66-97 DOI: $10.1159 / 000488001$
Windisch/Geiseler/Simon/Walterspacher/ Dreher 
standby for connection to a power source, thus allowing it to be put into use when necessary without endangering the patient.

\subsection{What Is the Procedure when a Ventilation}

Patient Undergoes Clinical Deterioration in the Home Setting?

Clinical deterioration of a patient requires medical consultation. In life-threatening situations, the appropriate emergency services should be alerted and adequate emergency measures should be implemented in the meantime; in all other situations, the treating outpatient physician (or his/her cover) should be called in (Ch. 6.6). In such situations, both the advanced health care directive and the fundamental therapy goals have to be taken into account (Ch. 16). The supervising HMV centre should be available to provide advice to physicians in the outpatient sector, to emergency physicians, or to admitting physicians in the hospital.

\subsection{In Which Cases Can the Extent of Nursing Care}

Be Reduced (So-Called "Reduction of Care")?

Reduction of care refers to a reduction in the extent of patient care carried out by the nursing staff. This is applicable to both specialised and assisted nursing care. The term "reduction of care" is not meant as a change in therapy goals that leans towards palliation.

Directly after being discharged into the outpatient ventilation sector for the first time, the patient often requires continuous care and supervision from nursing services or assisting personnel. After some time, an improvement in the general state of the patient, as well as the guidance of relatives and the patient itself, can lead to a reduction in this need, so that the undertaking of activities by the patient itself or his/her caregiver is possible. This can also positively contribute to the patient's sense of security. Therefore, the extent of care should always be oriented towards the patient's needs at the time as well as the individual circumstances. In principle, the aim should always be to achieve the greatest possible level of autonomy for the patient and his/her relatives.

The definitive implementation of reduction of care may only occur in consensus with the patient/legal guardian, the relatives, the multidisciplinary care team of nurses and therapists, and the registered physician responsible.

The following factors should be ensured for the reduction of care:

- The patient and his/her caregivers should demonstrate a pre-existing willingness for the reduction of care.

Treating Chronic Respiratory Failure with Invasive and Non-Invasive Ventilation
- Stable mechanical ventilation status.

- The securing of outpatient medical supervision and accessibility to a HMV centre.

- The securing of specialised, patient-appropriate treatment with consumables and aids; relatives and caregivers who are not officially qualified should be trained in their use.

- A stable family situation with the guarantee of safe implementation of the necessary treatment measures.

Care of the patient should also be ensured during the absence of qualified nursing staff. If necessary, individual, target-oriented training measures can be organised on site (Ch. 7).

Recommendation

- The indication for reduction of care must be made by the entire multidisciplinary team in conjunction with the patient concerned.

6.13 How Can Multidrug-Resistant Pathogens Be Dealt with in the Home Setting?

Along with antibiotic use, the continuous detection and evaluation of infections and pathogens is a legally bound process in clinical facilities (Infection protection law/\$23 Infektionsschutzgesetz). No such law exists for inpatient nursing facilities; however, based on a 2005 recommendation by the European Commission in collaboration with the Robert Koch Institute, the first point prevalence studies have been carried out in German nursing homes, providing information about the kinds of infections, pathogens, and antibiotic use that occur in such facilities [187-189]. Outpatient nursing home care has not yet been considered in this context, nor have shared residential communities, even though these may exhibit a similar risk profile to inpatient nursing facilities [190, 191].

The number and treatment details of HMV patients in Germany are still not known due to an inadequate depiction in the ICD catalogue and no available register. According to the experiences of this Guideline's experts, most of the patients undergoing invasive outpatient mechanical ventilation are treated in a communal intensive nursing care facility (Ch. 6). In general, these patients dealt with prolonged hospital stays in an intensive care unit, were treated in different hospitals (particularly those with weaning failure) and, in some cases, attended an early rehabilitation facility. For this reason, and because of the generally frequent antibiotic therapy for nosocomial infections, high usage rates of tracheal cannulae, permanent bladder catheters, and percutaneous feeding tubes, these patients have a high risk of colonisation with MRP. In outpatient communal intensive nursing care facilities, 
nursing staff simultaneously care for a number of patients per shift. Moreover, these carers often work in multiple facilities belonging to an enterprise and/or work on a freelance basis for different enterprises and might also work in clinical intensive care units. In all instances, the care providers are placed in a high-risk environment. On one hand, with inadequate hygiene measures comes a high risk of transmission, including for the other patients in the shared living facility, as well as for patients in other nursing facilities and intensive care units, if the transmission reaches the personnel. On the other hand, the measures for infection prevention are limited by the communal residents' entitlement to social care and quality of life: residents frequently share social rooms and participate in group activities together. Furthermore, the residents are mainly under the care of their own family doctor, making facility-spanning concepts for infection prevention difficult to establish, since regular communication between nursing staff and all independent physicians involved in the patient's treatment barely takes place.

The first hint that a significant problem with MRP exists in the outpatient intensive nursing care sector has been brought to light by an official survey in Munich: more than half (53\%) the residents of communal intensive nursing care facilities who underwent screening (screening rate, 78\%) were colonised with MRP [192]. The high MRP colonisation rate in communal intensive nursing care facilities may be traced back to the screening selection of severely ill, multimorbid patients who spent long periods in the intensive care unit. Despite the limitations of this telephone consultation-based study, there appears to be a substantial hygiene problem in the outpatient intensive nursing care sector; in particular, this increased prevalence may even have been underestimated due to the methodological approaches [192]. Therefore, this would mean that communal intensive nursing care facilities should be classified as hygiene risk areas; on one hand because of the high MRP colonisation rate, on the other because of the structurally high transmission risk and the markedly higher number of patients compared to the 1:1 carer-patient ratio in the home setting. Due to the fact that risk patients were selected for screening, the colonisation rates for gram-negative MRP (3MRGN and $4 \mathrm{MRGN}$ ) in communal intensive nursing care facilities appear to lie above the prevalence level reported for German intensive care units [193]. Hygiene planning and training for intensive nursing care staff would be desirable [194]. In the meantime, some German states have released recommendations for outpatient intensive nursing care.

\section{Nursing Qualifications for Home Mechanical Ventilation Care}

This chapter focuses on the issue of a needs-oriented qualification for nursing caregivers who are working with HMV individuals and aiming to provide a qualitatively high level of care. This qualification should essentially be oriented towards two distinct patient populations: (1) patients with physical disability and isolated respiratory failure, and (2) patients with a high number of comorbidities who require increased monitoring and nursing care.

7.1 What Sort of Challenges Should Be Taken into Account for Nursing Qualification Procedures in the Home Mechanical Ventilation Setting?

- Intensive care duties are carried out by the nursing team (e.g., in a shared living community), or independently by the single-acting nurse (1:1 care), without the presence of a physician.

- There are a multitude of heterogeneous diseases that lead to ventilation dependency, thus placing different demands on medical, nursing, therapeutic, and technical care services.

- There are many distinct forms of care (Ch. 6), for which the responsibilities are differentially regulated.

\subsection{What Sort of Special Knowledge and Skills Are \\ Relevant to Home Mechanical Ventilation Care?}

In the German HMV care sector, the highly complex tasks and active nursing care services are largely organised and carried out on an independent basis. A pronounced willingness to take on these responsibilities is therefore required. In addition to the specific nursing and therapeutic services, supporting and assisting the patient holds high significance, where the aim of this is to promote a mostly self-determined way of living and maximal participation in social activities. Moreover, the patient's relatives are often integrated into the care process, requiring good cooperation amongst caregivers [195].

The following knowledge and professional skills are specifically required for working in the outpatient intensive nursing care sector:

- Physiology of breathing and ventilation

- Diseases with respiratory failure

- Ventilation machine technology

- Monitoring/clinical observation

- Oxygen therapy

- Masks and tracheal cannulae, and their use

- Tracheostoma management
Windisch/Geiseler/Simon/Walterspacher/ Dreher 
- Methods for mobilisation and elimination of secretions

- Inhalation techniques

- Management of airway humidification

- Crisis management/emergency management

- Psychosocial support/promotion of social participation

- Hygiene management in the outpatient sector

\subsection{What Are the Special Requirements of the Nursing} Services and Facilities That Care for Ventilated

Patients?

Legal clauses in Germany do not include any particular licensing requirements for nursing services/nursing facilities that provide care for ventilated individuals $(\$ 132 \mathrm{a}$ Abs. 2 SGB V, \$72 SGB XI). Such special requirements would, however, be desirable in order to fairly meet the high demands associated with the care of these patients. Evaluation of licencing requirements requires research.

Due to the specific profile of demands placed on nursing staff, this Guideline recommends the following measures for quality control:

- Structured introductory training for new employees

- Enablement of an additional qualification for staff (basic course, Ch. 7.5; expert course, Ch. 7.4; nursing specialist for outpatient paediatric ventilation, Ch. 7.9)

- Refresher courses

- Professional support for nursing staff by employees with special expertise (division manager; see below and Ch. 7.4)

- Networking with each of the regional ventilation centres (Ch. 5.1) as well as with the privately registered physicians who specialise in the care of home-ventilated patients

In the ventilation sector, the facility must appoint specialised nurses as division managers. The number of appointed division managers in a facility should be oriented towards the number of patients requiring care. The division manager should be responsible for no more than 12 patients. Within the nursing sector, the division manager serves as a disseminator of knowledge; the division manager and nursing manager should not be one and the same person. Each ventilated patient and his/her relatives are informed of their appointed division manager.

In terms of its organisational responsibilities, the nursing service ensures that the specialised nurses designated to care for the ventilated patients receive proper introductory training and are installed according to their skills and knowledge. The nursing service should be reachable $24 \mathrm{~h}$ a day, 7 days a week [196].

Treating Chronic Respiratory Failure with Invasive and Non-Invasive Ventilation

\subsection{Who Is Eligible to Take on the Role of Division} Manager in a Nursing Service/Facility That Cares for Ventilated Patients?

Respiratory therapists are internationally involved in outpatient care of ventilated patients $[185,196]$. In Germany, respiratory therapists primarily work in a hospital or for mechanical ventilation companies, but less frequently in a nursing service or facility. For eligibility to work in an outpatient ventilation care facility for adult patients, a division manager should, in addition to the completion of a state-accredited qualification in a specialised nursing discipline (registered public health nurse, paediatric nurse, or geriatric nurse), be qualified in one of the following areas (for paediatrics, see Ch. 7.9):

- Respiratory therapist with nursing training

- Anaesthetics/intensive care nursing specialist

- Nursing specialist with at least 3 years' professional experience in the ventilation sector (intensive care unit, weaning unit, specialised ventilation unit, or HMV) within the last 5 years and successful completion of a certified expert course (a structured, extra-occupational training course with around 200 contact hours) for "Nursing care expert in outpatient ventilation"

Expert courses should be quality-assured through certification from professional societies and correspond at the very least to the requirements set down by the DIGAB e.V. (www.digab.de/weiterbildung).

The option to install nursing specialists with training in geriatric care is based on positive experiences indicating that - independent of the 3-year basic training course - the necessary competency for this purpose can be achieved through personal engagement and advanced training; this, however, needs to be confirmed through additional quality-controlled measures.

\subsection{What Are the Requirements of Nursing Specialists} Who Work as Part of a Nursing Service or in a Nursing Facility?

According to the SGB V, specialist nurses are appointed to the care of many different patients with distinct nursing, medical, therapeutic, and technical needs. They therefore require extensive training and the regular opportunity to undertake advanced training courses in order to maintain their skills and aptitude.

All nursing specialists amongst the nursing staff who work independently with ventilated patients (specialised nursing care) should, in addition to the completion of a state-accredited nursing specialisation (registered public health nurse, paediatric nurse, or geriatric nurse), be qualified in one of the following areas: 
- Respiratory therapist

- Anaesthetics/Intensive care nursing specialist

- Nursing specialist with at least 1 year of specific professional experience in the ventilation sector (intensive care unit, weaning unit, specialised ventilation unit, or HMV) within the last 5 years

- Nursing specialist with an additional qualification in the form of a successfully completed, certified expert training course (a structured, extra-occupational training course with around 200 contact hours) for "Nursing care expert in outpatient ventilation"

These basic courses should be quality-assured through certification by specialist societies and at the least correspond to the requirements set down by the DIGAB (www. digab.de/weiterbildung).

\subsection{Can Nursing Aids, Medical Assistants, Remedial}

Therapists, and Similar Professionals Participate in the Care of Ventilated Individuals?

Nursing aids, physician assistants, and remedial therapists may only work in the ventilation care branch as a part of a nursing team with nursing specialists (shared living communities or inpatient nursing facilities). Independent specialised nursing care of ventilated individuals is only possible with the qualifications described in Chapters 7.4. and 7.5. Following the relevant introductory training, these occupational groups can undertake basic nursing care work with ventilated patients (patient positioning, personal hygiene and grooming, patient transfer, nourishment, communication). Advanced training courses for this purpose should be developed in the future.

\section{Recommendations}

- Nursing specialists must complete the qualifying process with precise content and scope: at least one basic course with the qualification of "Nursing specialist for outpatient home mechanical ventilation."

- Nursing staff assigned to the care of ventilated patients must have (at least for every 12 patients) a division manager with additional qualifications in "respiratory therapy," "anaesthetics/intensive care nursing," or "Nursing expert in outpatient mechanical ventilation."

- Nursing aids, physician assistants, and remedial therapists may not work independently whilst caring for ventilated patients. Before potential undertaking of basic nursing duties within the nursing team, all persons from these occupational groups should receive introductory training and specific instruction.
7.7 What Are the Special Features of Assisted Care in the Context of a Care Support Model or an Employer Model?

The responsibility for the decision-making process associated with HMV care lies with the leading physician at the hospital, together with the patient and his/her caregivers. If the decision falls in favour of the care support model, the physician should inform the patient of the risks associated with receiving care from nursing staff who do not carry any formal qualifications. The patient should then be ready and able to take on this responsibility.

\subsubsection{Care Support Service}

When outpatient ventilation care occurs through aids who are employed by a nursing/assistant service, the responsibility for teaching and training lies with the nursing/assistant service (organisational responsibility of the employer) in close consultation with the patient concerned. In this respect, the personal capabilities of the patient are of great importance. It should be properly assessed which directions/instructions can be undertaken by the patient on an independent basis, and which should be organised by the nursing/assistant staff. Aids can also be in the form of registered carers without formal qualifications. The patient should provide documented approval for the selection of aid personnel.

Teaching measures for aid personnel should be developed in close collaboration with the patient, taking into account his/her individual needs and, when possible, be offered on site at the patient's place of residence in the form of "in-house training" [197]. Hereby, the content of Chapter 7.2 is thematically relevant, with special focus on therapy support and promoting patient independence.

\section{Recommendation}

- Aid personnel working as part of a nursing/assistant service should receive qualified training before commencing work with home mechanically ventilated patients, e.g., in the form of a basic course that abides with the minimum requirements of the DIGAB, or a similar alternative offer.

\subsubsection{Employer Model/Personal Budget}

HMV patients who wish to organise their own assisted care, independently of a commercial nursing/assistant service, can opt for the personal budget/employer model. Hereby, the patients take on the role of "employer" and knowingly accept the associated potential risks, in order to be able to determine their own lifestyle [198]. The selection of personal aids ("employees") and their introductory training and organisation of tasks should occur in- 
dependently and under the authority of the patient. Organisational competency is therefore an important prerequisite for this form of care. In cases of inpatient hospital treatment, aid personnel can support the patient and become integrated into the care program.

The extent of training and qualification required by aid personnel should correspond both to the patient's individual needs and the necessary level of support and care. This should be ascertained through discussion between the leading physician and the patient. The employer model places special demands on the patient itself, on one side as employer, on the other as instructor. Here, extra organisational support from the patient's family is particularly helpful. The skills required to attain instructional competency are often acquired through the patient's own initiative, but can also be supported through patient associations, self-help groups, and/or budget consultants. Based on the general experiences of this Guideline's expert panel, a mixed team comprising formally trained (registered) caregivers and informally trained aid personnel has proven to be a successful approach to the complex care program associated with HMV.

The enlistment of external experts with experience in the respective disease profile and the necessary therapeutic and nursing measures is recommended at least for the introductory training of aid personnel, but can also be useful in the further course of care. Measures for quality control are regulated within the personal budget objective agreements between the patient and the health insurance provider. To this end, uniform, content-related criteria for such quality control would be desirable.

With respect to the required quality control measures, aid personnel operating within the framework of the employer model/personal budget should also undergo training that is based on the criteria listed in Chapter 7.2. Therapy assistance and the support of patient autonomy should be the main topics of this training, where the subject-specific content of the basic course should also be taken into account (Ch. 7.5).

Recommendation

- For the care of mechanically ventilated patients carried out by aid personnel either from a commercial enterprise or within the realm of an employer model/personal budget model, formally and informally qualified aids should complete the appropriate training courses that fulfil the minimum requirements of the basic course (Ch. 7.5).

\subsection{How Can Relatives Be Prepared for the Nursing} Care of Ventilated Patients?

In cases where relatives are integrated into the care program, structured introductory training sessions occurring around, as well as directly with, the patient should take place early on, namely while the patient is still residing in the HMV centre [199-201]. Accordingly, a training program should be established $[196,200,202,203]$ that is repeatedly performed until each activity is mastered by the relatives. Ideally, this training should take place within short sessions of around 30 min duration [204]. The aim of these training measures is to acquire a definitive, individually tailored occupational competence so that proper care of the patient can be carried out.

For the implementation of relatives into the outpatient care of ventilated patients, enlistment of experts with experience and skill in the actual disease profile, and the application of necessary therapeutic measures, should be considered, at least for broadening the introductory training process, and for providing further support, if needed. The content of Chapter 7.2 is thematically relevant and can be adapted to the individual circumstances and needs of the patient. Relatives should be informed in a timely manner about the options available for easing the burden associated with this type of care (self-help groups, forums).

Recommendation

For the care of mechanically ventilated patients carried out by relatives, a needs-based, individually tailored introductory training program should be performed and documented early on in the discharging clinic (preferably a home mechanical ventilation centre).

\subsection{What Are the Special Qualification Requirements} for Nursing Specialists Caring for Children in the

\section{Home Mechanical Ventilation Setting?}

The regulations listed in Chapter 7.5 also pertain to specialised paediatric nursing care, along with some further adjustments that are necessary, in light of the special conditions associated with the care of children. Nursing specialists within a paediatric nursing service who work independently with mechanically ventilated children (specialised nursing care) should, in addition to the completion of a state-accredited nursing specialisation (registered public health/paediatric nurse; public health/paediatric nurse or geriatric nurse), demonstrate qualifications in one of the following specialities:

- Respiratory therapist with experience in paediatrics

- Paediatric nurse with specialisation in anaesthetics/intensive care 
- Nursing specialist with at least 1 year of area-specific work experience (paediatric ventilation sector) within the last 5 years

- Additional qualification through complete participation in a certified basic course (structured, extra-occupational advanced training course with at least 120 contact hours) in "Nursing specialisation in outpatient paediatric ventilation"

The basic course should be quality-assured through certification by specialist societies and, at the very least, correspond to the requirements set down by the DIGAB (www.digab.de/weiterbildung). Public health or geriatric nurses should only be implemented as a second choice. Geriatric nurses cannot take on the role of division manager (Ch. 15.6.3).

The option of using a geriatric nursing specialist in this situation is based on positive experiences showing that independent of the 3-year basic nursing training course - the level of competency required for this type of paediatric care can be acquired through personal engagement and requisite further training; this, however, should be confirmed through the additional application of appropriate quality control measures.

7.10 What Are the Special Qualification Requirements for Therapists Working in the Home Mechanical

Ventilation Sector?

Home-ventilated individuals often require extensive and supportive therapeutic measures to relieve discomfort, encourage independence, and improve movement and functional capabilities, which, in turn, can result in an enhanced quality of life. Physiotherapists, occupational therapists, and speech therapists working independently with ventilation-dependent patients should either have experience in the diseases at hand and their particular therapeutic implications or be trained accordingly. The aim in this field of work should also be to appoint therapists with sufficient occupational competency and specialised background knowledge, in order to be able to act in a satisfactory and proficient manner in cases of need. Specific quality control measures should therefore be developed through professional associations and specialist societies. The specific theoretical and practical content of a potential curriculum for therapists (ventilation therapy specialist) is currently being created as a pilot project in collaboration with the DIGAB.
7.11 What Are the Special Qualification Requirements for Physicians Working in the Home Mechanical Ventilation Sector?

The special requirements for physicians involved in outpatient care of ventilation patients are described in Chapter 6.6.

\subsection{Which Particular Topics Should Be Theoretically} and Practically Taught in the Advanced Qualification Course?

- Diseases with respiratory failure

- Gas exchange; differentiation between hypercapnic and hypoxic respiratory failure, and their development and therapy

- Different forms of mechanical ventilation including machine parameters; ventilator alarm functioning

- Invasive ventilation and NIV interfaces; functional check-ups; maintenance, care, and cleaning of cannulae and masks

- Cannula management; handling of cannula attachments; tracheal cannula exchange

- Tracheostomal management

- Operation, effectiveness, and handling of humidification systems

- Oxygen application, especially in combination with a ventilator; potential dangers of dealing with oxygen; risk of reduced respiratory drive during spontaneous breathing

- Indication for and implementation of the surveillance systems and interpretation of the results

- Secretion management, especially with respect to the particularities of the individual underlying diseases

- Criteria for monitoring spontaneous breathing periods prescribed by the physician

- Recognition of emergency situations and undertaking of emergency measures for mechanically ventilated patients

- Procedures for artificial feeding, including application systems and risks

- Recognition of swallowing disorders and aspiration

- Legally relevant stipulations (advanced health care directive, medical product law)

- Special requirements for the psychosocial support of long-term ventilated patients

- Hygiene in the home ventilation sector 


\section{Home Mechanical Ventilation following Weaning Failure}

\subsection{How Is Weaning Failure Defined?}

For ventilation withdrawal in the realm of intensive care-based ventilation therapy, three different weaning categories that follow international standards can be defined: Category 1 - simple weaning; Category 2 - difficult weaning; Category 3 - prolonged weaning [205]. Patients who receive NIV therapy after weaning are defined here as "weaning in progress," while "non-weanability" from invasive ventilation is rarely mentioned. To this end, data from German studies show that among patients who were relocated to a specialised weaning centre with suspected weaning failure, only around 38\% actually had definitive weaning failure with subsequent invasive HMV therapy [206]. Similar figures have emerged from Great Britain [207]. To better depict the reality of care and to emphasise the high significance of NIV in the weaning process, the German S2k Guideline "Prolonged Weaning" additionally distinguishes between the following categories: Category $3 \mathrm{a}$ - "prolonged weaning without the need for NIV"; Category $3 \mathrm{~b}$ - "prolonged weaning requiring subsequent NIV," and Category $3 c$ - "definitive weaning failure with subsequent invasive HMV or death" (Table 6) [152]. Furthermore, there is an increasing number of patients with tracheal cannulae who are nonetheless released from hospital or a rehabilitation facility without ventilatory support; these patients are, however, not discussed in this Guideline.

Unlike the elective initiation of invasive HMV in chronic respiratory failure patients, weaning failure is concerned with persistent respiratory failure arising from an acute or acute-to-chronic disease. Category $3 \mathrm{~b}$ is not rated as weaning failure, since the mainly intermittent NIV therapy can be predominantly carried out on an independent basis at home. In contrast to this, invasive HMV requires substantial personnel and technical support and is associated with a marked loss of the patient's independence $[152,208]$. The indication for continuing invasive ventilation after weaning failure should be regularly reviewed by experts after its initial implementation, preferably in a HMV centre (Ch. 5.1.) [152, 207, 209]. It is especially important to frequently evaluate the option of transferring to NIV, since a mid- to long-term improvement in muscle strength or swallowing function and hence weaning potential - is attained in quite a few patients through regular physiotherapy and speech therapy, respectively [210]. In the event of persistent weaning failure, the continuation of ventilation therapy should be balanced out with the patient's wishes.

Treating Chronic Respiratory Failure with Invasive and Non-Invasive Ventilation
Recommendation

A diagnosis of "definitive weaning failure" (Category 3c) must be made by a physician (ideally from a specialised weaning centre) with experience in mechanical ventilation and weaning, or should at least be assessed via consultation before invasive home mechanical ventilation due to definitive weaning failure is initiated.

\subsection{Is Weaning Failure Reversible?}

Typical reasons for (i) the existence of weaning potential after primary weaning failure and (ii) the transition to invasive HMV are listed as follows:

- Muscle weakness following long-term intensive care therapy (intensive care unit-acquired weakness) [211, 212]

- Ventilator-induced diaphragmatic dysfunction [153, $213,214]$

- Stabilisation of comorbidities

- Improvement of a swallowing disorder

For this reason, the continuation of intensive physiotherapy and speech therapy and, if necessary, occupational therapy is indicated in the outpatient sector for patients with primary weaning failure.

\subsection{How Should the Diagnosis of Weaning Failure Be Made?}

In general, all avenues must be exhausted to avoid invasive HMV. The diagnosis of weaning failure should only be attested by a specialised weaning centre (Ch. 5.1). In particular, whether the transfer from invasive to non-invasive mechanical ventilation is feasible must be evaluated before initial discharge with invasive mechanical ventilation into the home setting [152, 184, 196]. Given the wide-reaching consequences for patients, relatives, and the shared-risk community within the general health care system, the valid diagnosis of definitive weaning failure (assessed at least via consultation, e.g., in the realm of an expert panel) by a medical specialist from a specialised weaning centre must precede invasive HMV.

The American Guideline for weaning from mechanical ventilation recommends establishing the diagnosis weaning failure only after 3 months of unsuccessful weaning treatment [215]. In the current German Guideline for prolonged weaning from mechanical ventilation, experts conclude that specialised weaning centres can often gauge after just 4 weeks whether the continuation of weaning is worthwhile [152].

If the diagnosis of definitive weaning failure has been correctly made, this indicates that no weaning potential remained at the time of discharge. Due to a lack of scien- 
tific evidence, and for reasons of patient safety, further weaning attempts should be avoided in the home setting, since the weaning process often requires invasive diagnostic and therapeutic measures, which are generally not available in the outpatient sector.

Discharging a patient into invasive HMV primarily for the purpose of ventilation weaning is obsolete $[152,181]$; however, active care and goal-directed continuation of supportive therapeutic measures can facilitate the acquisition of future weaning potential.

\subsection{How Is the Indication for Invasive Mechanical Ventilation Established in the Event of Definitive Weaning Failure?}

The indication for invasive HMV in patients with definitive weaning failure can only be made when a secure home care environment has been established. Additionally, a life perspective should be taken into account that corresponds to the patient's circumstances (Ch. 6.1). There is no indication for invasive HMV if it prolongs the dying process [10, 196, 216-222]. Sometimes the severity of the disease (including the corresponding medical, nursing, therapeutic, and technical needs) limits the desire for release into the home sector (Ch. 6.3).

The prerequisite for the initiation of invasive HMV due to definitive weaning failure is the consent from the patient and/or his/her legal guardian. To this end, and in light of the current German legislation (patient rights law from February 20, 2013), the patient and/or legal guardian must be extensively informed about the living and specialised care circumstances expected under invasive HMV conditions, with detailed information of the individual possibilities versus limitations.

In particular, given the severe underlying multiple morbidities that often accompany patients with definitive weaning failure, firm discussion about the prognosis for survival is required. This includes the achievable level of quality of life with respect to the patient's own wishes, and the aspired level of independence.

Valid consent for this form of therapy can only be obtained after a thorough clarification process. In patients that are unable to provide their consent and do not possess an advanced health care directive, the presumed wishes of the patient have to be determined just like in any other therapy decision.

The prerequisite for the initiation of invasive home ventilation in patients with definitive weaning failure can only be fulfilled if the medical and care situations are stable and there is adequate control of symptoms. There should be no need for the frequent presence of a physician or for repeated medical interventions in the form of diagnostic and/or therapeutic measures (Ch. 6.3) [152, 181].

End-of-life patients can, if they wish, be discharged from hospital with invasive mechanical ventilation, provided appropriate palliative medical care can be applied in the outpatient setting. In the meantime, specialised outpatient palliative care has also become available in Germany for non-oncological patients, and can be prescribed in addition to nursing care according to the SGB V.

However, not every specialised outpatient palliative care team is familiar with the modalities of HMV. Hospice care would be desirable for some patients but is not yet widely available, partly due to varying opinions about the use of mechanical ventilation at the end-of-life stage.

Before discharging a patient with invasive HMV into the home sector, the curative or palliative medical therapy goals of the outpatient care need to be defined. They should be documented along with the content of an advanced health care directive (if available) in the letter of discharge.

Quality Indicator

- Invasive home mechanical ventilation is only established after consent is given by the extensively informed patient and/or his/her legal guardian. The content of the information is documented and signed by the informing doctor and patient/legal guardian. In this context, determining the patient's wishes is documented in a comprehensible fashion.

Recommendations

- The indication for invasive home mechanical ventilation after definitive weaning failure must not only be based on medical facts (the need for continuing invasive ventilation) but also on ethical aspects.

- The individual wishes of the thoroughly informed patient must be taken into account for the therapy decision-making process.

- To determine the indication for invasive home mechanical ventilation in cases where the patient is not able to provide consent, an inquiry into the patient's wishes - if possible through the undertaking of an ethical case conference - must be comprehensively documented.

\subsection{Where Should Invasive Home Mechanical}

\section{Ventilation Be Initiated following Weaning Failure?}

Through the long-term care of patients undergoing non-invasive and, to a lesser extent, invasive HMV for chronic respiratory failure, ventilation centres have at hand the required knowledge and understanding of the outpatient care system and options. The infrastructural requirements for certified weaning clinics - such as those of the DGP - comprise the existence of a specialised ward 
for HMV where patients, relatives, and nursing staff can be appropriately briefed and where HMV and its specific features can be prepared. In addition, these procedural Guidelines comprise a patient-focused management scheme as well as recommendations for check-up examinations, thus allowing the issues of patient safety and long-term care to be adequately addressed. Established spinal cord transection and paediatric clinics also have a similar infrastructure. If possible, the initiation of invasive HMV for definitive weaning failure following intensive therapy should take place in a specialised weaning centre, or in a HMV centre with expertise in invasive HMV (Ch. 5.1) [10, 183, 185, 203, 208, 209, 223, 224]. If relocation of the patient is not possible, such a centre should at least be involved in the valid establishment of the indication as well as the transfer process and aftercare of the patient, with appropriate follow-up examinations and consultation services.

\section{Quality Indicator}

- The initiation of invasive home mechanical ventilation for patients with definitive weaning failure takes place in - or at least in collaboration with - a specialised weaning centre or in a home mechanical ventilation centre with expertise in invasive home mechanical ventilation. The discharging centre, or the centre participating in the discharging process, is available to the home therapy team for consultation.

\section{Recommendations}

- Invasive home mechanical ventilation should be initiated in a specialised weaning centre or a home mechanical ventilation centre with expertise in invasive home mechanical ventilation (Ch. 5.1); at the very least, this process must take place via consultation with such a centre.

- If a patient with invasive ventilation is released from a clinic that is not a ventilation centre, a ventilation expert from such a specialised centre must be enlisted within the shortest time period (maximum 3 months). Alternatively, an assessment of weaning potential and the home care situation should be undertaken in consultation with a specialised ventilation centre by an authorised/privately registered physician with expertise in mechanical ventilation. Absorption of costs by the cost bearer for domestic health care must be limited up until this time point.

8.6 What Should Be Noted for the Outpatient Medical Care of Patients with Weaning Failure?

When initiating invasive HMV due to definitive weaning failure, the underlying complex of multiple morbidities should especially be taken into account, since this requires adequate medical care in the home setting $[10,181$, 196, 203, 225, 226]. Besides pharmaceutical and nonpharmaceutical therapy, the coordination of additionally important therapy measures (such as physiotherapy, speech therapy, occupational therapy, wound management, and pain management) should be supplemented with medical knowledge about the prognosis and weaning potential of the patient. The medical qualifications that are required for dealing with invasively ventilated patients are described in Chapter 6.6.

Beyond the valid, expert-based diagnosis of definitive weaning failure, the medical prerequisites for safe release of the patient into the outpatient sector are:

- Haemodynamic stability without the use of vasoactive medication.

- Absence of acute hyper- or hypoactive delirium

- Stable renal function or the securing of outpatient renal dialysis therapy, including the availability of a dialysis unit

- Existence of a nutrition plan, including the potentially necessary application devices (PEG, Port, Boroviac catheter)

- A safe medication plan that can be used and prescribed within the outpatient sector

- A lack of demand for regular immobilisation, blood sampling, imaging, or invasive interventions/therapy measures [181, 227]

Before discharging the patient into the outpatient sector, a screening test for MRP must be carried out and the result immediately reported to the intended care facility. Appropriate hygiene measures based on legal specifications and institutional recommendations are to be arranged between the discharging hospital and the outpatient care team.

Even the provision of medical care to multimorbid elderly people in German inpatient nursing facilities is still regarded as inadequate [228]. Since the necessary extent of expertise in mechanical ventilation cannot be provided by privately registered physicians, and the elaborate care program with home visits is either not at all - or only poorly - represented within the reimbursement system [229], the outpatient medical care of multimorbid patients receiving invasive $\mathrm{HMV}$ is, according to the experiences of this Guideline's expert panel, largely not guaranteed. As a result, outpatient care circumstances are often unstable to the point where the frequent deployment of emergency doctors and repeated hospital admissions are necessary. Outpatient intensive care nursing services therefore collaborate frequently with self-employed intensive care physicians additionally working in hospitals. For the doctors concerned, such a collaboration can potentially be problematic on social, civil, occupational, liability, and fiscal levels, especially when they are commissioned by the outpatient care services and paid from the 
home health care funds (SGB V). These informal, legally non-conforming configurations should be redeemed either through the creation of appropriate intersectoral care concepts (e.g., integrated care plans, selective agreements) or the authorisation of eligible hospital physicians; this hereby requires urgent action. Currently, only one type of collaboration (SGB XI) between inpatient nursing facilities and hospitals is legally possible.

A problematic observation is that despite the exhaustive use of personnel-based or technical help, quality of life continues to be poor after definitive weaning failure, especially in COPD patients. To this end, the measurement of health-related quality of life through the application of questionnaires (e.g., the ventilation-specific SRI questionnaire; for further information, see https://www. pneumologie.de/service/patienteninformation/patienten-fragebogen-zur-befindlichkeit-bei-schwerer-respiratorischer-insuffizienz/) can be considered (Huttmann S, Storre J, Windisch W, 2017, pers. commun.).

For invasively ventilated patients with complex multiple morbidities, home doctor visits are imperative to minimise logistically complex and hazardous transports by vehicle. The ventilation centre responsible for the longterm supervision of the patient should therefore be available for consultation with the private treating physician. In the future, the telemedicine branch might be able to support these consulting sessions through offers such as teleconsultations [230]. This kind of communication network between private treating physicians and ventilation centres might also facilitate early detection of further weaning potential that has developed in patients despite their primary definitive weaning failure. In the event of advanced clinical deterioration under outpatient ventilation conditions, the patient's care situation as well as therapy goals should be assessed.

Quality Indicators

- The provision of medical care in the outpatient sector is secured through home doctor visits.

- The ventilation centre responsible for the long-term supervision of the patient is available for consultation with the treating physician.

Recommendations

- After first implementing invasive home mechanical ventilation due to definitive weaning failure, check-up examinations must take place at least once a year in a specialised ventilation centre.

- The therapy goals of patients with invasive home mechanical ventilation due to definitive weaning failure must be regularly reviewed by a physician.

\section{Acknowledgment}

We thank Dr. Sandra Dieni for her assistance in translating the manuscript.

\section{References}

For references, see online supplementary material (for all online suppl.material, seewww.karger.com/doi/10.1159/000488001).

\begin{tabular}{ll} 
Abbreviations used in this paper \\
\hline AHCD & Advanced health care directive \\
ALS & Amyotrophic lateral sclerosis \\
AWMF & $\begin{array}{l}\text { Arbeitsgemeinschaft der Wissenschaftlichen Medizinischen Fachgesellschaften e.V. (Association } \\
\text { of the Scientific Medical Societies in Germany) }\end{array}$ \\
BGA & Blood gas analysis \\
CCHS & Congenital central hypoventilation syndrome/Ondine's curse \\
CHS & Central hypoventilation syndrome \\
CIM & Critical illness myopathy \\
CIP & Critical illness polyneuropathy \\
CO 2 & Carbon dioxide \\
COPD & Chronic obstructive pulmonary disease \\
CPAP & Continuous positive airway pressure \\
DGP & Deutsche Gesellschaft für Pneumologie und Ventilationsmedizin e.V. (German Respiratory \\
DIGAB & Society) \\
& Deutsche interdisziplinäre Gesellschaft für außerklinische Beatmung e.V. (German \\
ECG & Interdisciplinary Society for Home Mechanical Ventilation) \\
EPAP & Electrocardiogram \\
\hline
\end{tabular}




\section{Abbreviations used in this paper}

$\mathrm{FEV}_{1}$

FVC

HME

HMV

IPPB

KRINKO

Forced expiratory volume in 1 second

Forced vital capacity

Heat and moisture exchange

Home mechanical ventilation

Intermittent positive pressure breathing

LTOT

MDI

MI-E

MRP

NIV

NMD

$\mathrm{O}_{2}$

Kommission für Krankenhaushygiene und Infektionsprävention (Commission for Hygiene in

Hospital and the Prevention of Infection)

Long-term oxygen treatment

Metered-dose inhaler

Mechanical Insufflator-Exsufflator

Multidrug-resistant pathogen

Non-invasive ventilation

Neuromuscular diseases

OHS

OSAS

$\mathrm{PaCO}_{2}$

$\mathrm{PaO}_{2}$

$\mathrm{PCF}$

$\mathrm{PCO}_{2}$

PEEP

$\mathrm{PO}_{2}$

$\mathrm{PtcCO}_{2}$

REM

RERA

SGB

SMI

Oxygen

Obesity hypoventilation syndrome

Obstructive sleep apnoea syndrome

Partial pressure of arterial carbon dioxide

Partial pressure of arterial oxygen

Peak cough flow

Partial pressure of carbon dioxide

Positive end-expiratory pressure

Partial pressure of oxygen

Partial pressure of transcutaneous carbon dioxide

Rapid eye movement

Respiratory effort-related arousal

Sozialgesetzbuch (Social Security Code)

$\mathrm{SpO}_{2} \quad$ Oxygen saturation

SRI Severe Respiratory Insufficiency Questionnaire

VC Vital capacity 